\title{
An equivariant index for proper actions II: properties and applications
}

\author{
Peter Hochs* and Yanli Song ${ }^{\dagger}$
}

September 23, 2021

\begin{abstract}
In the first part of this series, we defined an equivariant index without assuming the group acting or the orbit space of the action to be compact. This allowed us to generalise an index of deformed Dirac operators, defined for compact groups by Braverman. In this paper, we investigate properties and applications of this index. We prove that it has an induction property that can be used to deduce various other properties of the index. In the case of compact orbit spaces, we show how it is related to the analytic assembly map from the BaumConnes conjecture, and an index used by Mathai and Zhang. We use the index to define a notion of K-homological Dirac induction, and show that, under conditions, it satisfies the quantisation commutes with reduction principle.
\end{abstract}

\section{Contents}

1 Introduction 2

2 Preliminaries 4

2.1 The G-index . . . . . . . . . . . . . . . 4

2.2 Deformed Dirac operators . . . . . . . . . . 6

2.3 Dirac induction ................... 7

*University of Adelaide, peter. hochs@adelaide. edu . au

${ }^{\dagger}$ University of Toronto, songyanl@math. utoronto.ca 
3 Induction from slices $\quad 9$

3.1 The induction result $\ldots \ldots \ldots \ldots$

3.2 Compact groups and deformed Dirac operators . . . . . . 11

3.3 A special case ... . . . . . . . . . . . 15

3.4 An explicit form of the Morita equivalence isomorphism . . 17

3.5 Product metrics . . . . . . . . . . . . . . . . 19

3.6 Morita equivalence and the G-index . . . . . . . . . 20

4 Properties of the G-index 23

4.1 The analytic assembly map and the Mathai-Zhang index . . 23

4.2 Another relation with the assembly map . . . . . . . . 28

4.3 K-homological Dirac induction ............ 29

4.4 Spin $^{c}$-quantisation commutes with reduction ....... 34

\section{A Notation and conventions}

\section{Introduction}

In part I of this series, an equivariant index was developed that applies to actions by possibly noncompact groups, and with possibly noncompact orbit spaces. To recall the definition of this index, we let $\mathrm{G}$ be an almost connected Lie group (i.e. having finitely many connected components), acting properly and isometrically on a Riemannian manifold $M$. Let $\mathcal{E}=\mathcal{E}^{+} \oplus \mathcal{E}^{-}$ be a $\mathbb{Z}_{2}$-graded, Hermitian, G-equivariant vector bundle. Let $\mathrm{D}$ be an odd, self-adjoint, G-equivariant, first order differential operator on $\mathcal{E}$. If $M$ and $\mathrm{G}$ are compact, then we have the usual equivariant index

$$
\operatorname{index}_{G}(D):=\left[\operatorname{ker} D^{+}\right]-\left[\operatorname{ker} D^{-}\right] \quad \in R(G),
$$

where $D^{ \pm}$is the restriction of $D$ to $\Gamma^{\infty}\left(\mathcal{E}^{ \pm}\right)$, and $R(G)$ is the representation ring of $\mathrm{G}$.

The definition of the equivariant index has been generalised to cases where either the orbit space $M / G$ or the group $G$ is compact. If $M / G$ is compact, then one has the analytic assembly map from the Baum-Connes conjecture [6] for elliptic operators, and Kasparov's index of transversally elliptic operators [24]. If $\mathrm{G}$ is compact, then Braverman [9] defined an index of a natural class of deformed Dirac operators. This, and equivalent indices, has been used very successfully in geometric quantisation [20, 27, 29].

The techniques used in the cases where $\mathrm{M} / \mathrm{G}$ or $\mathrm{G}$ are compact are very different. If $M / G$ is compact, one can use operator algebraic techniques 


\begin{tabular}{|c|c|c|}
\hline & $\begin{array}{l}\text { M/G compact, } \\
\text { D transversally elliptic }\end{array}$ & $\begin{array}{l}\text { M/G noncompact, } \\
\text { D a deformed Dirac operator }\end{array}$ \\
\hline G compact & Atiyah, 1974 [4] & Braverman, 2002 [9] \\
\hline G noncompact & Kasparov, 2015 [24] & Part I, 2016 [21] \\
\hline
\end{tabular}

Table 1: Special cases of the G-index

to obtain an index in the K-theory or K-homology of a $\mathrm{C}^{*}$-algebra related to the group $\mathrm{G}$. If $\mathrm{G}$ is compact, it is natural to define an index in the completed representation ring

$$
\hat{\mathrm{R}}(\mathrm{G}):=\left\{\bigoplus_{\pi \in \hat{\mathrm{G}}} \mathrm{m}_{\pi} \pi ; \mathrm{m}_{\pi} \in \mathbb{Z}\right\} .
$$

Because of the different approaches in the two cases, it is not immediately clear how to construct a common generalisation, i.e. an equivariant index that can be used when both $\mathrm{M} / \mathrm{G}$ and $\mathrm{G}$ are noncompact. This was done in [21], where the condition of D being G-Fredholm was introduced, which implies that D has a G-index

$$
\operatorname{index}_{G}(D) \in K K\left(C_{0}(G / K) \rtimes G, \mathbb{C}\right) .
$$

Here $\mathrm{K}<\mathrm{G}$ is a maximal compact subgroup. This $\mathrm{K}$-homology group of the crossed product $C_{0}(G / K) \rtimes G$ can be identified with $\hat{R}(K)$ via the Morita equivalence $C_{0}(G / K) \rtimes G \sim C^{*} K$. The main result in [21] is that a natural class of deformed Dirac operators is G-Fredholm. This completes Table 1 . by filling in the bottom-right entry. As far as the authors are aware, the $\mathrm{G}$-index is the first equivariant index that applies in cases where both $\mathrm{M} / \mathrm{G}$ and $\mathrm{G}$ are noncompact. Here by an equivariant index, we mean an index taking values in an object defined purely in terms of the group acting.

In the present paper, we study properties of the G-index of deformed Dirac operators. We start by proving an induction property of the index. This is an explicit description of the image of the G-index in $\hat{R}(K)$ in terms of data on a global, $K$-invariant slice $N \subset M$ such that $M=G \times_{K} N$. Besides giving a better understanding of the G-index, the induction property can also be used to prove various other properties of it.

One such property is a relation with the analytic assembly map from the Baum-Connes conjecture [6] if $M / G$ is compact. If $G$ is semisimple with discrete series representations, then it turns out that the assembly map can be recovered directly from the G-index. Another application of the 
induction result is a quantisation commutes with reduction property of the Gindex. This generalises the main result in [20], where $G$ was assumed to be compact.

Independently of the induction result, we give a second relation between the G-index and the assembly map, and an index defined by Mathai and Zhang [28], if $M / G$ is compact. But our main interest is in cases where both $M / G$ and $G$ are noncompact, which are furthest removed from existing index theory. One example of this setting is the action by $G$ on $T^{*}(G / K)$. In that case, we use the G-index to define a version of the Dirac induction isomorphism from the Connes-Kasparov conjecture, which is now defined on $\hat{R}(K)$.

\section{Acknowledgements}

The authors thank Maxim Braverman, Nigel Higson, Gennadi Kasparov, Mathai Varghese and Guoliang Yu for interesting and helpful discussions. The first author was supported by the European Union, through Marie Curie fellowship PIOF-GA-2011-299300.

\section{Preliminaries}

Let $\mathrm{G}$ be an almost connected Lie group, i.e. having finitely many connected components. We recall the definition of G-Fredholm operators and their Gindices, as introduced in part I of this series [21]. We also state the main result from [21], that deformed Dirac operators are G-Fredholm. Finally, we recall the notion of Dirac induction used in the Connes-Kasparov conjecture, which will be used in some of the applications.

\subsection{The G-index}

For the rest of this paper, we fix a maximal compact subgroup $K<G$, and a proper, isometric action by $G$ on a Riemannian manifold $M$. Let $\mathcal{E}=\mathcal{E}^{+} \oplus \mathcal{E}^{-} \rightarrow M$ be a $\mathbb{Z}_{2}$-graded, Hermitian vector bundle. Suppose the action by $\mathrm{G}$ lifts to $\mathcal{E}$, preserving the grading and the Hermitian metric.

Since the action is proper, Abels' theorem [1] guarantees the existence of a smooth, equivariant map

$$
p: M \rightarrow G / K .
$$


Equivalently, we have a G-equivariant diffeomorphism

$$
\mathrm{M} \cong \mathrm{G} \times{ }_{\mathrm{K}} \mathrm{N}
$$

via the action map, with $N:=p^{-1}(e K)$. Pullback along $p$ defines a Gequivarant map

$$
p^{*}: C_{0}(G / K) \rightarrow C_{b}(M) .
$$

This induces a $*$-homomorphism

$$
p_{G}^{*}: C_{0}(G / K) \rtimes G \rightarrow C_{b}(M) \rtimes G
$$

between crossed-product $C^{*}$-algebras [34].

The representation by $C_{b}(M)$ in $L^{2}(\varepsilon)$ by pointwise multiplication, and the unitary representation of $\mathrm{G}$ in $\mathrm{L}^{2}(\mathcal{E})$ combine to a representation

$$
\pi_{\mathrm{G}, \mathrm{C}_{\mathrm{b}}(M)}: \mathrm{C}_{\mathrm{b}}(\mathrm{M}) \rtimes \mathrm{G} \rightarrow \mathcal{B}\left(\mathrm{L}^{2}(\mathcal{E})\right) .
$$

Conider the representation

$$
\pi_{\mathrm{G}, \mathrm{G} / \mathrm{K}}^{\mathrm{p}}:=\pi_{\mathrm{G}, \mathrm{C}_{\mathrm{b}}(\mathrm{M})} \circ \mathrm{p}_{\mathrm{G}}^{*}: \mathrm{C}_{0}(\mathrm{G} / \mathrm{K}) \rtimes \mathrm{G} \rightarrow \mathcal{B}\left(\mathrm{L}^{2}(\mathcal{E})\right) .
$$

It is explicitly given by

$$
\left(\pi_{\mathrm{G}, \mathrm{G} / \mathrm{K}}^{\mathrm{p}}(\varphi) \mathrm{s}\right)(\mathrm{m})=\int_{\mathrm{G}} \varphi(\mathrm{g}, \mathrm{p}(\mathrm{m})) \mathrm{g} \cdot\left(\mathrm{s}\left(\mathrm{g}^{-1} \mathrm{~m}\right)\right) \mathrm{dg},
$$

for $\varphi \in \mathrm{C}_{\mathrm{c}}\left(\mathrm{G}, \mathrm{C}_{0}(\mathrm{G} / \mathrm{K})\right), \mathrm{s} \in \mathrm{L}^{2}(\mathcal{E})$ and $\mathrm{m} \in \mathrm{M}$.

Let $\mathrm{D}$ be an odd, self-adjoint, G-equivariant, first order differential operator on $\mathcal{E}$. In [21], the operator D was defined to be G-Fredholm for $p$ if the triple

$$
\left(\mathrm{L}^{2}(\varepsilon), \frac{\mathrm{D}}{\sqrt{\mathrm{D}^{2}+1}}, \pi_{\mathrm{G}, \mathrm{G} / \mathrm{K}}^{\mathrm{p}}\right)
$$

is a Kasparov $\left(C_{0}(G / K) \rtimes G, \mathbb{C}\right)$ module. Then the $G$-index of $D$ is defined as the class

$$
\operatorname{index}_{G}^{p}(D) \in K K\left(C_{0}(G / K) \rtimes G, \mathbb{C}\right)
$$

of (2.2). Via the Morita equivalence $C_{0}(G / K) \rtimes G \sim C^{*} K$, this index can be identified with an element of

$$
\mathrm{KK}\left(\mathrm{C}^{*} \mathrm{~K}, \mathbb{C}\right)=\hat{\mathrm{R}}(\mathrm{K}):=\left\{\bigoplus_{\pi \in \widehat{\mathrm{R}}} \mathrm{m}_{\pi} \pi ; \mathrm{m}_{\pi} \in \mathbb{Z}\right\} .
$$


If $\mathrm{D}$ is G-Fredholm for any map $\mathrm{p}$ as above, then it is called G-Fredholm. Its G-index is then independent of $p$ (see Lemma 3.2 in [21]), and denoted by $\operatorname{index}_{G}(D)$.

If $D$ is transversally elliptic in the sense of Definition 6.1 in [24], and $\mathrm{M} / \mathrm{G}$ is compact, then $\mathrm{D}$ is G-Fredholm by Proposition 6.4 and Remark 8.19 in [24]. The G-index of D is then a generalisation of Atiyah's index of elliptic operators [4] to noncompact groups.

Another class of G-Fredholm operators is obtained by applying a natural deformation to Dirac-type operators.

\subsection{Deformed Dirac operators}

Suppose that $M$ is complete in the given Riemannian metric. Let

$$
c: \operatorname{TM} \rightarrow \operatorname{End}(\varepsilon)
$$

be a vector bunde endomorphism into the odd endomorphisms, such that for all $v \in \mathrm{TM}$,

$$
c(v)^{2}=-\|v\|^{2} .
$$

Suppose that $\mathrm{c}(\mathrm{g} \cdot v)=\mathrm{g} \circ \mathrm{c}(v) \circ \mathrm{g}^{-1}$ for all $\mathrm{g} \in \mathrm{G}$ and $v \in \mathrm{TM}$.

Let $\nabla^{\mathcal{E}}$ be a G-invariant Hermitian connection on $\mathcal{E}$, such that for all vector fields $v, w \in X(M)$,

$$
\left[\nabla_{v}^{\mathcal{E}}, \mathrm{c}(w)\right]=\mathrm{c}\left(\nabla_{v}^{\mathrm{TM}} w\right)
$$

where $\nabla^{\mathrm{TM}}$ is the Levi-Civita connection of the Riemannian metric. Then we have the Dirac operator

$$
\mathrm{D}:=\mathrm{c} \circ \nabla^{\mathcal{E}}: \Gamma^{\infty}(\mathcal{\varepsilon}) \rightarrow \Gamma^{\infty}(\mathcal{\varepsilon}) .
$$

Let $\psi: M \rightarrow \mathfrak{g}$ be a G-equivariant smooth map, with respect to the adjoint action by $\mathrm{G}$ on the Lie algebra $\mathfrak{g}$. This map defines a vector field $v^{\psi} \in X(M)$ by

$$
v_{\mathrm{m}}^{\psi}:=\left.\frac{\mathrm{d}}{\mathrm{dt}}\right|_{\mathrm{t}=0} \exp (-\mathrm{t} \psi(\mathrm{m})) \cdot \mathrm{m},
$$

for all $m \in M$. A key assumption we make is that the set Zeroes $\left(v^{\psi}\right) \subset M$ of zeroes of $v^{\psi}$ is cocompact, i.e. Zeroes $\left(v^{\psi}\right) / \mathrm{G}$ is compact. The Dirac operator deformed by $\psi$ is the operator

$$
\mathrm{D}_{\psi}:=\mathrm{D}-\sqrt{-1} \mathrm{c}\left(v^{\psi}\right) .
$$


Given a real-valued function $\rho \in \mathrm{C}^{\infty}(M)^{\mathrm{G}}$, we call a nonnegative function $\mathrm{f} \in \mathrm{C}^{\infty}(\mathrm{M})^{\mathrm{G}} \rho$-admissible if

$$
\frac{f}{\|d f\|+f+1} \geq \rho
$$

Such functions exist for all $\rho$, see Lemma 3.10 in [21].

Suppose for now that $\mathrm{G}=\mathrm{K}$ is compact. A re-interpretation of Theorem 2.9 in [9] is that there is a function $\rho \in C^{\infty}(M)^{k}$, such that for all $\rho$-admissible functions $f$, the operator $D_{f \psi}$ is K-Fredholm. Then its index

$$
\operatorname{index}_{K}\left(D_{f \psi}\right) \in K K\left(C^{*} K, \mathbb{C}\right)=\widehat{R}(K)
$$

is the index studied in [9] (see Lemma 2.9 in [21]). It is independent of $f$ and $\nabla^{\mathcal{E}}$.

The main result in [21] is that this generalises to noncompact groups.

Theorem 2.1. There is a function $\rho_{\mathrm{G}} \in \mathrm{C}^{\infty}(\mathrm{M})^{\mathrm{G}}$ such that for all $\rho$-admissible functions $\mathrm{f}$, the operator $\mathrm{D}_{\mathrm{f \psi}}$ is $\mathrm{G}$-Fredholm for $\mathrm{p}$.

See Theorem 3.12 in [21]. Furthermore, the G-index of $D_{f \psi}$ is independent of $p, f, \nabla^{\mathcal{E}}$, and the Riemannian metric on TM, as stated precisely in Proposition 3.13 in [21].

In this paper, we study properties of the G-index of deformed Dirac operators, and relations with the analytic assembly map [6].

\subsection{Dirac induction}

In some of the applications, we will use the Dirac induction isomorphism from the Connes-Kasparov conjecture. We recall the definition of this isomorphism here.

Fix a K-invariant inner product on the Lie algebra $\mathfrak{g}$, and let $\mathfrak{p} \subset \mathfrak{g}$ be the orthogonal complement to $\mathfrak{k}$. Suppose that the representation

$$
\text { Ad: } \mathrm{K} \rightarrow \mathrm{SO}(\mathfrak{p})
$$

lifts to a homomorphism

$$
\widetilde{\mathrm{Ad}}: \mathrm{K} \rightarrow \operatorname{Spin}(\mathfrak{p}) .
$$

This lift always exists if one replaces $G$ by a double cover. (See the end of this subsection for the case where it does not exist.) Let $S_{\mathfrak{p}}$ be the spinor representation of $\operatorname{Spin}(\mathfrak{p})$, see e.g. Definition 5.11 in [26]. We view $S_{\mathfrak{p}}$ as a 
representation of $K$, via the map $\widetilde{A d}$. If $\mathfrak{p}$ is even-dimensional, then $S_{\mathfrak{p}}$ has a natural $\mathbb{Z}_{2}$-grading $S_{\mathfrak{p}}=S_{\mathfrak{p}}^{+} \oplus S_{\mathfrak{p}}^{-}$. By the element $\left[S_{\mathfrak{p}}\right] \in R(K)$, we will mean $\left[S_{\mathfrak{p}}^{+}\right]-\left[S_{\mathfrak{p}}^{-}\right]$in that case.

Existence of the lift (2.3) is equivalent to $\mathrm{G} / \mathrm{K}$ having a G-equivariant Spin-structure, with spinor bundle $G \times{ }_{K} S_{\mathfrak{p}} \rightarrow G / K$. We will then say that $\mathrm{G} / \mathrm{K}$ is equivariantly Spin.

For any finite-dimensional representation space $V$ of $K$, we have the Gequivariant vector bundle

$$
\mathcal{E}_{\mathrm{V}}:=\mathrm{G} \times_{\mathrm{K}}\left(\mathrm{S}_{\mathfrak{p}} \otimes \mathrm{V}\right) \rightarrow \mathrm{G} / \mathrm{K}
$$

Let $\left\{X_{1}, \ldots, X_{\operatorname{dim} \mathfrak{p}}\right\}$ be an orthonormal basis of $\mathfrak{p}$. Consider the Dirac operator

$$
D_{V}:=\sum_{j=1}^{\operatorname{dim} \mathfrak{p}} X_{j} \otimes c\left(X_{j}\right) \otimes 1_{V}
$$

on

$$
\Gamma^{\infty}\left(\mathcal{E}_{\mathrm{V}}\right) \cong\left(\mathrm{C}^{\infty}(\mathrm{G}) \otimes \mathrm{S}_{\mathfrak{p}} \otimes \mathrm{V}\right)^{\mathrm{K}} .
$$

Here $c$ denotes the Clifford action by $\mathfrak{p}$ on $S_{\mathfrak{p}}$. It defines an equivariant K-homology class

$$
\left[\mathrm{D}_{\mathrm{V}}\right] \in \mathrm{KK}_{*}^{\mathrm{G}}\left(\mathrm{C}_{0}(\mathrm{G} / \mathrm{K}), \mathbb{C}\right) .
$$

The analytic assembly map from the Baum-Connes conjecture [6, 22] is a map

$$
\mu_{Y}^{\mathrm{G}}: \mathrm{KK}_{*}^{\mathrm{G}}\left(\mathrm{C}_{0}(\mathrm{Y}), \mathbb{C}\right) \rightarrow \mathrm{KK}_{*}\left(\mathbb{C}, \mathrm{C}_{\mathrm{r}}^{*} \mathrm{G}\right)
$$

for any cocompact, proper $\mathrm{G}$-space $\mathrm{Y}$. Here $\mathrm{C}_{\mathrm{r}}^{*} \mathrm{G}$ is now the reduced group $\mathrm{C}^{*}$-algebra of $\mathrm{G}$. There is also a version for the maximal group $\mathrm{C}^{*}$-algebra. Applying this map for $Y=G / K$ to the class (2.5) yields

$$
\mu_{G / K}^{G}\left[D_{V}\right] \in K K\left(\mathbb{C}, C_{r}^{*} G\right) \text {. }
$$

The Dirac induction map

$$
\mathrm{D}-\mathrm{Ind}_{\mathrm{K}}^{\mathrm{G}}: \mathrm{R}(\mathrm{K}) \rightarrow \mathrm{KK}_{*}\left(\mathbb{C}, \mathrm{C}_{\mathrm{r}}^{*} \mathrm{G}\right)
$$

is defined by $[\mathrm{V}] \mapsto \mu_{\mathrm{G} / \mathrm{K}}^{\mathrm{G}}\left[\mathrm{D}_{\mathrm{V}}\right]$, for finite-dimensional representation spaces $\mathrm{V}$ of $\mathrm{K}$. It maps into even KK-theory if $\mathrm{G} / \mathrm{K}$ is even-dimensional, and into odd KK-theory otherwise. The Connes-Kasparov conjecture, proved by Chabert, Echterhoff and Nest [14], states that it is in isomorphism of Abelian groups. (This was proved for linear reductive groups by Wassermann [33].) 
By the universal coefficient theorem, we have

$$
\mathrm{KK}_{*}\left(\mathrm{C}_{\mathrm{r}}^{*} \mathrm{G}, \mathbb{C}\right) \cong \operatorname{Hom}_{\mathbb{Z}}\left(\mathrm{KK}_{*}\left(\mathbb{C}, \mathrm{C}_{\mathrm{r}}^{*} \mathrm{G}\right), \mathbb{Z}\right)
$$

via the Kasparov product. Pulling back along Dirac induction therefore defines an isomorphism of Abelian groups

$$
\left(\mathrm{D}-\operatorname{Ind}_{\mathrm{K}}^{\mathrm{G}}\right)^{*}: \mathrm{KK}\left(\mathrm{C}_{\mathrm{r}}^{*} \mathrm{G}, \mathbb{C}\right) \stackrel{\cong}{\rightrightarrows} \widehat{\mathrm{R}}(\mathrm{K}) \text {. }
$$

If the lift (2.3) does not exist, i.e. $\mathrm{G} / \mathrm{K}$ is not equivariantly Spin, one still has a Dirac induction isomorphism. Let $\pi: \tilde{G} \rightarrow G$ be a double cover for which (2.3) exists, and let $\tilde{K}:=\pi^{-1}(K)$. Let $u$ be the nontrivial element of $\operatorname{ker} \pi$. Set

$$
R_{\text {Spin }}(K):=\left\{V \in R(\tilde{K}) ; u \text { acts trivially on } V \otimes S_{\mathfrak{p}}\right\}
$$

Then for $V \in R_{\text {Spin }}(K)$, the tensor product $V \otimes S_{\mathfrak{p}}$ can be viewed as a (virtual) representation of $K$, and the above constructions apply. This yields an isomorphism

$$
\text { D-Ind } \mathrm{K}_{\mathrm{K}}^{\mathrm{G}}: \mathrm{R}_{\text {Spin }}(\mathrm{K}) \stackrel{\cong}{\cong} \mathrm{KK}_{*}\left(\mathbb{C}, \mathrm{C}_{\mathrm{r}}^{*} \mathrm{G}\right) \text {. }
$$

\section{Induction from slices}

We have seen in Theorem 2.1 that deformed Dirac operators have welldefined G-indices. In the rest of this paper, we discuss properties of these indices. One useful tool is the induction result we prove in this section, Theorem 3.2. It is a relation between the G-index of a deformed Dirac operator on $M$, and the $K$-index of an operator on a slice $N \subset M$. The latter index can be described explicitly in terms of the $\mathrm{L}^{2}$-kernel of the operator.

We keep using the notation and assumptions of Subsection 2.2. We fix a smooth, equivariant map $p: M \rightarrow G / K$, and consider the corresponding slice $\mathrm{N}=\mathrm{p}^{-1}(\mathrm{eK})$.

\subsection{The induction result}

Consider the restriction $\left.\nabla^{\mathcal{E}}\right|_{\mathrm{N}}$ of $\nabla^{\mathcal{E}}$ to $\mathrm{N}$. Since $\left.\mathrm{TN} \subset \mathrm{TM}\right|_{\mathrm{N}}$, we have the Clifford action

$$
c_{N}:\left.\left.\mathbb{N} \otimes \mathcal{E}\right|_{N} \rightarrow \mathcal{E}\right|_{N} .
$$

These combine to the Dirac-type operator

$$
\mathrm{D}^{\mathrm{N}}: \Gamma^{\infty}\left(\left.\mathcal{E}\right|_{\mathrm{N}}\right) \stackrel{\left.\nabla^{\mathcal{E}}\right|_{\mathrm{N}}}{\longrightarrow} \Gamma^{\infty}\left(\left.\mathrm{TN} \otimes \mathcal{E}\right|_{\mathrm{N}}\right) \stackrel{\mathrm{c}_{N}}{\longrightarrow} \Gamma^{\infty}\left(\left.\mathcal{E}\right|_{\mathrm{N}}\right) .
$$


As before, we fix a K-invariant inner product on $\mathfrak{g}$. Let $\mathfrak{p} \subset \mathfrak{g}$ be the orthogonal complement to $\mathfrak{k}$. Then

$$
\mathfrak{g}=\mathfrak{k} \oplus \mathfrak{p} .
$$

We will identify

$$
\mathrm{TN} \oplus \mathrm{N} \times\left.\mathfrak{p} \cong \mathrm{TM}\right|_{\mathrm{N}}
$$

via the map

$$
(v,(n, X)) \mapsto v+X_{n}^{M}
$$

for $n \in N, v \in T_{n} N$ and $X \in \mathfrak{p}$.

Let $\mathrm{B}$ be the given Riemannian metric on $M$. We will consider two Kinvariant metrics on $\left.T M\right|_{N}$. One is simply the restriction $\left.B\right|_{N}$. The other is defined by the properties that the decomposition (3.3) is orthogonal, the metric equals $\left.B\right|_{\mathbb{N} N}$ on $\mathbb{T N}$, and is defined by the inner product on $\mathfrak{g}$ on $\mathrm{N} \times \mathfrak{p}$. We denote this second metric by $B_{\mathfrak{p}}$.

Choose a K-equivariant, isometric vector bundle isomorphism

$$
\left(\left.\mathrm{TM}\right|_{N}, B_{\mathfrak{p}}\right) \rightarrow\left(\left.\mathrm{TM}\right|_{N},\left.B\right|_{N}\right),
$$

which is the identity on TN. Via this map, the Clifford action

$$
\left.c\right|_{N}:\left(\left.T M\right|_{N},\left.B\right|_{N}\right) \rightarrow \operatorname{End}(\varepsilon)
$$

defines the Clifford action

$$
c_{\mathfrak{p}}:\left(\left.T M\right|_{N}, B_{\mathfrak{p}}\right) \rightarrow \operatorname{End}(\mathcal{E}) .
$$

We have $\left.c_{\mathfrak{p}}\right|_{\mathrm{TN}}=\left.c\right|_{\mathrm{TN}}$.

For a real-valued function $f \in C^{\infty}(M)^{G}=C^{\infty}(N)^{K}$, consider the deformed Dirac operator

$$
D_{\left.f \psi\right|_{N}}^{N}=D^{N}-\sqrt{-1} c_{p}\left(\left.v^{f \psi}\right|_{N}\right)
$$

on $\Gamma^{\infty}\left(\left.\mathcal{E}\right|_{N}\right)$. Under the additional assumption that $\psi(N) \subset \mathfrak{k}$, this is the Dirac operator on $\left.\mathcal{E}\right|_{N}$ deformed by $\left.f \psi\right|_{N}$ as in Subsection 2.2, but we do not make this assumption. (See Subsection 3.3 for other consequences of that assumption.) The analogue of Theorem 2.1 still holds, however.

Proposition 3.1. There is a positive function $\rho_{N} \in C^{\infty}(N)^{K}=C^{\infty}(M)^{G}$ such that if $\mathrm{f}$ is $\rho_{\mathrm{N}}$-admissible, then $\mathrm{D}_{\left.\mathrm{f} \psi\right|_{\mathrm{N}}}^{\mathrm{N}}$ is K-Fredholm. 
This proposition is not a direct consequence of Theorem 2.1. since the vector field $\left.v^{\psi}\right|_{\mathrm{N}}$ is not induced by a map $\mathrm{N} \rightarrow \mathfrak{k}$ unless $\psi(\mathrm{N}) \subset \mathfrak{k}$. For this reason, and to illustrate a simpler approach that is possible for compact groups, we give a separate proof of Proposition 3.1 in Subsection 3.2. This is also a simpler proof of Theorem 2.1 in the case where $\mathrm{G}$ is compact. Furthermore, in the case of trivial groups, the arguments in Subsection 3.2 yield a criterion for Callias-type operators to be Fredholm.

Another way to prove Proposition 3.1 would have been to slightly generalise the proof of Proposition 3.15 in [21]. Indeed, write $\psi=\psi_{\mathfrak{k}} \oplus \psi_{\mathfrak{p}}$ according to the decomposition (3.2). Then if one adds a sixth term

$$
A_{6}:=-\sqrt{-1} \sum_{j=1}^{\operatorname{dim} N} c\left(e_{j}\right) c\left(\nabla_{e_{j}}^{\mathbb{T N}} f v^{\psi_{p}}\right)
$$

(where $\left\{e_{1}, \ldots, e_{\operatorname{dim} N}\right\}$ is a local orthonormal frame for $\mathrm{TN}$, and $\nabla^{\mathrm{TN}}$ is the Levi-Civita connection for the restricted Riemannian metric on TN) in Lemma 4.6 and Proposition 5.2 in [21], one obtains the estimates necessary to prove a version of Proposition 3.15 in [21] that implies Proposition 3.1.

Let $\rho_{\mathrm{N}}$ be as in Proposition 3.1. and suppose $f$ is $\rho_{\mathrm{N}}$-admissible. Then by Lemma 2.9 in [21], we have

$\operatorname{index}_{K}\left(D_{\left.f \psi\right|_{N}}^{N}\right)=\left[\operatorname{ker}_{L^{2}}\left(D_{\left.f \psi\right|_{N}}^{N}\right)^{+}\right]-\left[\operatorname{ker}_{L^{2}}\left(D_{\left.f \psi\right|_{N}}^{N}\right)^{-}\right] \in K K\left(C^{*} K, \mathbb{C}\right) \cong \widehat{R}(K)$.

The induction result relates this index to the $G$-index $\operatorname{index}_{G}(\mathcal{E}, \psi)$ of $D_{f \psi}$, which makes the latter more concrete and computable.

Theorem 3.2 (Induction from slices). The multiplicity of every irreducible representation of $\mathrm{K}$ in the $\mathrm{L}^{2}$-kernel of $\mathrm{D}_{\left.\mathrm{f} \psi\right|_{\mathrm{N}}}^{\mathrm{N}}$ is finite. Under the identification $\mathrm{KK}\left(\mathrm{C}_{0}(\mathrm{G} / \mathrm{K}) \rtimes\right.$ $\mathrm{G}, \mathbb{C})=\widehat{\mathrm{R}}(\mathrm{K})$ by Morita equivalence, we have

$$
\operatorname{index}_{G}(\mathcal{E}, \psi)=\left[\operatorname{ker}_{\mathrm{L}^{2}}\left(\mathrm{D}_{\left.\mathrm{f} \psi\right|_{\mathrm{N}}}^{\mathrm{N}}\right)^{+}\right]-\left[\operatorname{ker}_{\mathrm{L}^{2}}\left(\mathrm{D}_{\left.\mathrm{f} \psi\right|_{\mathrm{N}}}^{\mathrm{N}}\right)^{-}\right] \in \widehat{R}(\mathrm{~K}) .
$$

\subsection{Compact groups and deformed Dirac operators}

We will prove a slightly more general statement than Proposition 3.1. Consider the setting of Subsection 3.1. Suppose $\psi(N) \subset \mathfrak{k}$. (What follows will later be applied to the component $\psi_{\mathfrak{k}}$ of $\psi$ in $\mathfrak{k}$.) Let a nonnegative function $f \in C^{\infty}(N)^{K}$ be given. Let $T \in \operatorname{End}\left(\left.\varepsilon\right|_{N}\right)^{K}$ be a fibrewise self-adjoint, odd vector bundle endomorphism, such that

$$
c\left(v^{\psi}\right) \mathrm{T}+\mathrm{Tc}\left(v^{\psi}\right)=0,
$$


and

$$
\mathrm{D}^{\mathrm{N}} \mathrm{fT}+\mathrm{fTD}^{\mathrm{N}} \in \operatorname{End}\left(\left.\varepsilon\right|_{\mathrm{N}}\right) .
$$

Suppose that the pointwise norm of the endomorphism $(3.6)$ is bounded above by

$$
\Theta \cdot(\|d f\|+f)
$$

for a function $\Theta \in C^{\infty}(N)^{K}$ (independent of $f$ ). Also suppose that the endomorphism $\left\|v^{\psi}\right\|^{2}+\mathrm{T}^{2}$ of $\left.\mathcal{E}\right|_{\mathrm{N}}$ is invertible outside a compact set. For $f \in C^{\infty}(N)^{K}$, we consider the operator

$$
D_{f \psi}^{T}=D_{f \psi}^{N}+f T=D^{N}+f\left(-\sqrt{-1} c\left(v^{\psi}\right)+T\right) .
$$

on $\Gamma^{\infty}\left(\left.\varepsilon\right|_{N}\right)$. This is a combination of a deformed Dirac operator as studied in this paper, and a Callias-type operator [3, 11, 12, 13, 25].

Proposition 3.3. There is a positive function $\rho_{\mathrm{N}} \in \mathrm{C}^{\infty}(\mathrm{N})^{\mathrm{K}}$ such that if $\mathrm{f}$ is $\rho_{\mathrm{N}}$-admissible, then the operator $\mathrm{D}_{\mathrm{f \psi}}^{\mathrm{T}}$ is $\mathrm{K}$-Fredholm.

Remark 3.4. If $K$ is the trivial group, so that $\psi=0$, and if $T^{2}$ is invertible outside a compact set, then Proposition 3.3 shows that the Callias-type operator

$$
D_{f}^{T}=D^{N}+f \cdot T
$$

is Fredholm on the noncompact manifold $N$, for admissible functions $f$.

To deduce Proposition 3.1 from Proposition 3.3, write $\psi=\psi_{\mathfrak{k}} \oplus \psi_{\mathfrak{p}}$ according to the decomposition (3.2). Then if we replace $\psi$ by $\psi_{\mathfrak{k}}$ in Proposition 3.3, and set $T:=\sqrt{-1} c_{\mathfrak{p}}\left(v^{\psi_{\mathfrak{p}}}\right)$, then the conditions (3.5) and (3.6) hold, so Proposition 3.1 follows. The least trivial conditions to check are the following.

Lemma 3.5. The operators $\mathrm{c}\left(v^{\psi_{\mathfrak{k}}}\right)$ and $\mathrm{c}_{\mathfrak{p}}\left(v^{\psi_{\mathfrak{p}}}\right)$ anticommute, and

$$
\mathrm{D}^{\mathrm{N}} \mathrm{fc}_{\mathfrak{p}}\left(v^{\psi_{\mathfrak{p}}}\right)+\mathrm{fc}_{\mathfrak{p}}\left(v^{\psi_{\mathfrak{p}}}\right) \mathrm{D}^{\mathrm{N}}
$$

is a vector bundle endomorphism of $\left.\mathcal{E}\right|_{\mathrm{N}}$.

Proof. The claim follows from the fact that for the metric $B_{\mathfrak{p}}$ on $\left.T M\right|_{N}$, tangent vectors to $\mathrm{N}$ are orthogonal to tangent vectors defined by elements of $\mathfrak{p}$. This immediately implies that $c\left(v^{\psi_{\mathfrak{k}}}\right)$ and $\mathrm{c}_{\mathfrak{p}}\left(v^{\psi_{\mathfrak{p}}}\right)$ anticommute. It also implies that, in terms of a local orthonormal frame $\left\{e_{1}, \ldots, e_{\operatorname{dim} N}\right\}$ of $\mathrm{TN}$,

$$
D^{N} f c_{\mathfrak{p}}\left(v^{\psi_{\mathfrak{p}}}\right)+f c_{\mathfrak{p}}\left(v^{\psi_{\mathfrak{p}}}\right) D^{N}=\sum_{j=1}^{\operatorname{dim} N} c\left(\nabla_{e_{j}}^{T M} f v^{\psi_{\mathfrak{p}}}\right),
$$

so the claim follows. 
Let $\mathfrak{t} \subset \mathfrak{k}$ be a maximal torus. Fix a set of positive roots for $\left(\mathfrak{k}_{\mathbb{C}}, \mathfrak{t}_{\mathbb{C}}\right)$. Let $\rho_{K}$ be half the sum of these positive roots (not to be confused with the function $\rho$ as in Theorem 2.1). Let $\Lambda_{+} \subset \sqrt{-1} t^{*}$ be the set of dominant integral weights. For $\lambda \in \Lambda_{+}$, let $V_{\lambda}$ be the irreducible representation space of $K$ with highest weight $\lambda$. For $\lambda \in \Lambda_{+}$, let $L^{2}\left(\left.\varepsilon\right|_{N}\right)_{\lambda}$ be the $V_{\lambda}$-isotypical component of $\mathrm{L}^{2}\left(\left.\mathcal{E}\right|_{\mathrm{N}}\right)$.

Lemma 3.6. There is a real-valued function $\rho_{\mathrm{N}} \in \mathrm{C}^{\infty}(\mathrm{N})^{\mathrm{K}}$, such that if $\mathrm{f}$ is $\rho_{\mathrm{N}}$-admissible, then for all $\lambda \in \Lambda_{+}$, and all $\mathrm{a}_{\lambda}>0$, the operator

$$
\left.\left(\left(D_{f \psi}^{\top}\right)^{2}+a_{\lambda}\right)^{-1}\right|_{L^{2}\left(\left.\varepsilon\right|_{N}\right)_{\lambda}}
$$

on $\mathrm{L}^{2}\left(\left.\mathcal{E}\right|_{\mathrm{N}}\right)_{\lambda}$ is compact.

Proof. Analogously to Lemma 4.5 in [21], we have the local expression, with respect to a local orthonormal frame $\left\{e_{1}, \ldots, e_{\operatorname{dim} N}\right\}$ of $\mathrm{TN}$,

$$
\begin{aligned}
& \left(D_{f \psi}^{T}\right)^{2}= \\
& \left(D^{N}\right)^{2}+f^{2}\left(\left\|v^{\psi}\right\|^{2}+T^{2}\right)+\sqrt{-1} \sum_{j=1}^{\operatorname{dim} N} c\left(e_{j}\right) c\left(\nabla_{e_{j}}^{T M} f v^{\psi}\right)-2 \sqrt{-1} f \nabla_{v^{\psi}}^{\left.\mathcal{E}\right|_{N}}+\left(D^{N} f T+f T D^{N}\right) .
\end{aligned}
$$

By assumption on $T$, there is a function $\Theta_{1} \in C^{\infty}(N)^{K}$ (independent of $f$ and $\lambda$ ) such that we have the pointwise estimate

$$
\left\|\sqrt{-1} \sum_{j=1}^{\operatorname{dim} N} c\left(e_{j}\right) c\left(\nabla_{e_{j}}^{\mathrm{TM}} f v^{\psi}\right)+\left(D^{N} f \mathrm{~T}+\mathrm{fTD}^{\mathrm{N}}\right)\right\| \leq \Theta_{1}(\|\mathrm{df}\|+\mathrm{f})
$$

Let $C_{\lambda}>0$ be such that for all $X \in \mathfrak{k}$, the operator on $V_{\lambda}$ defined by $X$ has norm at most $C_{\lambda}\|X\|$. For every $n \in N$, the operator

$$
\nabla_{v_{n}^{\psi}}^{\left.\mathcal{E}\right|_{N}}-\mathcal{L}_{\psi(n)}
$$

is a linear endomorphism of $\varepsilon_{n}$. Since, for such $n$, we have

$$
\left.\mathcal{L}_{\psi(\mathrm{n})}\right|_{\mathrm{L}^{2}(\varepsilon)_{\lambda}} \leq \mathrm{C}_{\lambda}\|\psi(\mathrm{n})\|,
$$

there is a positive function $\Theta_{2} \in C^{\infty}(N)^{K}$ (independent of $f$ and $\lambda$ ) such that

$$
\left|\nabla_{v^{\psi}}^{\left.\mathcal{E}\right|_{\mathrm{N}}}\right| \leq \Theta_{2}+\mathrm{C}_{\lambda}\|\psi\|
$$

where use the absolute value of operators as before. 
We conclude that

$$
\left.\left(D_{f \psi}^{T}\right)^{2}\right|_{L^{2}\left(\left.\mathcal{E}\right|_{N}\right)_{\lambda}} \geq\left.\left(D^{N}\right)^{2}\right|_{L^{2}\left(\left.\mathcal{E}\right|_{N}\right)_{\lambda}}+f^{2}\left(\left\|v^{\psi}\right\|^{2}+T^{2}\right)-\left(\Theta_{1}+\Theta_{2}+C_{\lambda}\|\psi\|\right)(\|d f\|+f)
$$

Let $\tilde{\rho}_{N} \in C^{\infty}(N)^{K}$ be a real-valued function such that $\left(\Theta_{1}+\Theta_{2}\right)\left(\tilde{\rho}_{N}-1\right)$ tends to infinity faster than $\|\psi\|$ as its argument tends to infinity. Choose $\rho_{\mathrm{N}} \in \mathrm{C}^{\infty}(\mathrm{N})^{\mathrm{K}}$ such that, outside a relatively compact neighbourhood of the points $n \in N$ where $\left\|v_{n}^{\psi}\right\|^{2}+\left\|T_{n}\right\|^{2}=0$, we have

$$
\rho_{\mathrm{N}} \geq \frac{\left(\Theta_{1}+\Theta_{2}\right) \tilde{\rho}_{\mathrm{N}}}{\left\|v^{\psi}\right\|^{2}+\|\mathrm{T}\|^{2}}
$$

In addition, choose $\rho_{N}$ so that it is at least equal to 1 outside a compact set. Suppose $f$ is $\rho_{N}$-admissible. Then by (3.7), we find that

$$
\left.\left(D_{f \psi}^{T}\right)^{2}\right|_{L^{2}\left(\left.\mathcal{E}\right|_{N}\right)_{\lambda}} \geq\left.\left(D^{N}\right)^{2}\right|_{L^{2}\left(\left.\mathcal{E}\right|_{N}\right)_{\lambda}}+\zeta_{\lambda}
$$

where $\zeta_{\lambda} \in \mathrm{C}^{\infty}(\mathrm{N})^{\mathrm{K}}$ satisfies

$$
\zeta_{\lambda} \geq\left(\left(\Theta_{1}+\Theta_{2}\right)\left(\tilde{\rho}_{N}-1\right)-C_{\lambda}\|\psi\|\right)(\|d f\|+f),
$$

outside a relatively compact neighbourhood of the set $\left\{n \in N ;\left\|v_{n}^{\psi}\right\|^{2}+\right.$ $\left.\left\|T_{n}\right\|^{2}=0\right\}$. Since $f \geq 1$ outside a compact set, and by the assumption on $\tilde{\rho}_{N}$, the function on the right hand side tends to infinity as its argument tends to infinity. This implies that $\left.\left(D_{f \psi}^{T}\right)^{2}\right|_{L^{2}\left(\left.\varepsilon\right|_{N}\right)_{\lambda}}$ has discrete spectrum. It follows that

$$
\left(\left(D_{f \psi}^{\top}\right)^{2}+a_{\lambda}\right)^{-1}
$$

is indeed a compact operator on $\mathrm{L}^{2}\left(\left.\varepsilon\right|_{N}\right)_{\lambda}$, for all $a_{\lambda}>0$.

In the proof of Proposition 3.3, we will use the Casimir element $\Omega_{\mathrm{K}}$ in the centre of the universal enveloping algebra of $\mathfrak{k}$. For $\lambda \in \Lambda_{+}$, the element $\Omega_{\mathrm{K}}$ acts on $V_{\lambda}$ as the scalar

$$
\left\|\lambda+\rho_{K}\right\|-\left\|\rho_{K}\right\|
$$

(These norms are defined by the same inner product that was used to define $\Omega_{K}$, the one fixed before to define the metric $B_{\mathfrak{p}}$.)

The operator $\left(D_{f \psi}^{T}\right)^{2}+1$ is invertible by Proposition 10.2.11 in [15]. Since $\Omega_{K}$ is a nonnegative operator, the operator $\left(D_{f \psi}^{T}\right)^{2}+\Omega_{K}+1$ is invertible as well. The main part of the proof of Proposition 3.3 is the following. 
Lemma 3.7. There is a real-valued function $\rho_{\mathrm{N}} \in \mathrm{C}^{\infty}(\mathrm{N})^{\mathrm{K}}$, such that if $\mathrm{f}$ is $\rho_{\mathrm{N}}$-admissible, the operator

$$
\left(\left(D_{f \psi}^{T}\right)^{2}+\Omega_{K}+1\right)^{-1}
$$

on $\mathrm{L}^{2}\left(\left.\mathcal{E}\right|_{\mathrm{N}}\right)$ is compact.

Proof. For $\lambda \in \Lambda_{+}$, the Casimir operator $\Omega_{K}$ acts on $L^{2}\left(\left.\varepsilon\right|_{N}\right)_{\lambda}$ as the scalar (3.8). By K-equivariance of $D_{f \psi}^{\top}$, we therefore have the decomposition

$$
\left(\left(D_{\mathrm{f} \psi}^{T}\right)^{2}+\Omega_{\mathrm{K}}+1\right)^{-1}=\bigoplus_{\lambda \in \Lambda_{+}}\left(\left.\left(D_{\mathrm{f} \psi}^{\mathrm{T}}\right)^{2}\right|_{\mathrm{L}^{2}\left(\left.\varepsilon\right|_{N}\right)_{\lambda}}+\left\|\lambda+\rho_{\mathrm{K}}\right\|-\left\|\rho_{\mathrm{K}}\right\|+1\right)^{-1} .
$$

By Lemma 3.6 every term in this sum is compact. Since the norm of a direct sum of operators is the supremum of the norms of the terms, the above direct sum converges in the operator norm, to a compact operator.

Proof of Proposition 3.3. Let $e \in \mathrm{C}^{\infty}(\mathrm{K})$. Since $\Omega_{\mathrm{K}}$ commutes with $\mathrm{D}^{\mathrm{N}}, \mathrm{T}$ and $c\left(v^{\psi}\right)$, we have

$$
\begin{aligned}
\pi_{K}(e)\left(\left(D_{f \psi}^{\top}\right)^{2}+1\right)^{-1}-\pi_{K}(e)\left(\left(D_{f \psi}^{\top}\right)^{2}+\Omega_{K}^{r}+1\right)^{-1} \\
=-\left(\left(D_{f \psi}^{\top}\right)^{2}+1\right)^{-1} \Omega_{K} \pi_{K}(e)\left(\left(D_{f \psi}^{\top}\right)^{2}+\Omega_{K}^{r}+1\right)^{-1},
\end{aligned}
$$

as a simpler analogue of Lemma 4.6 in [21]. Because $\Omega_{K} \pi_{K}(e)=\pi_{K}\left(\Omega_{K} e\right)$ is a bounded operator, Lemma 3.7 implies that

$$
\pi_{\mathrm{K}}(e)\left(\left(\mathrm{D}_{\mathrm{f \psi}}^{\mathrm{T}}\right)^{2}+1\right)^{-1}
$$

is a compact operator, which implies the claim.

\subsection{A special case}

Under some additional assumptions, Theorem 3.2 takes a simpler form. One of these assumptions is that $\psi(N) \subset \mathfrak{k}$. This assumption is not satisfied in all relevant examples, see Subsection 4.3. In fact, cases where this assumption is not satisfied are furthest removed from existing index theory, and therefore potentially the most interesting.

Since we now suppose that $\psi(N) \subset \mathfrak{k}$, the operator $D_{\left.f \psi\right|_{N}}^{N}$ in Theorem 3.2 is precisely of the form studied by Braverman in [9]. Therefore, Braverman's cobordism invariance result, Theorem 3.7 in [9], and all of its consequences, generalise to the G-index of deformed Dirac operators, under this additional assumption. 
Suppose, furthermore, that $\mathrm{G} / \mathrm{K}$ is even-dimensional, and equivariantly Spin, with $\mathbb{Z}_{2}$-graded spinor bundle

$$
\mathcal{E}_{\mathrm{G} / \mathrm{K}}=\mathrm{G} \times{ }_{\mathrm{K}} \mathrm{S}_{\mathfrak{p}} .
$$

Let $\mathrm{Cl}(\mathrm{N} \times \mathfrak{p})=\mathrm{N} \times \mathrm{Cl}(\mathfrak{p})$ be the Clifford bundle of $\mathrm{N} \times \mathfrak{p} \rightarrow \mathrm{N}$. Consider the $\mathrm{Cl}(\mathrm{N} \times \mathfrak{p})$-module $\mathrm{N} \times \mathrm{S}_{\mathfrak{p}}$, and the Clifford module

$$
\mathcal{E}_{\mathrm{N}}:=\operatorname{Hom}_{\mathrm{Cl}(\mathrm{N} \times \mathfrak{p})}\left(\mathrm{N} \times \mathrm{S}_{\mathfrak{p}}, \mathcal{E}\right) .
$$

Then, since $S_{\mathfrak{p}}$ is an irreducible representation of $\mathrm{Cl}(\mathfrak{p})$,

$$
\mathcal{E}=\mathcal{E}_{\mathrm{N}} \otimes S_{\mathfrak{p}}
$$

Let $D^{\varepsilon_{N}}$ be the Dirac operator associated to any K-invariant Clifford connection on $\varepsilon_{N}$. Since now the vector bundle endomorphism $c_{\mathfrak{p}}\left(\left.v^{\mathrm{f} \psi}\right|_{\mathrm{N}}\right)$ of $\left.\mathcal{E}\right|_{N}$ acts trivially on the factor $N \times S_{\mathfrak{p}}$, we have the deformed Dirac operator

$$
\mathrm{D}_{\mathrm{f \psi}}^{\mathcal{E}_{\mathrm{N}}}:=\mathrm{D}^{\mathcal{E}_{\mathrm{N}}}-\sqrt{-1} \mathrm{c}_{\mathfrak{p}}\left(\left.v^{\mathrm{f \psi}}\right|_{\mathrm{N}}\right)
$$

on $\Gamma^{\infty}\left(\varepsilon_{N}\right)$. In terms of the decomposition (3.11, we then have

$$
\mathrm{D}_{\left.\mathrm{f} \psi\right|_{\mathrm{N}}}^{\mathrm{N}}=\mathrm{D}_{\mathrm{f} \psi}^{\mathcal{E}_{\mathrm{N}}} \otimes 1_{S_{\mathfrak{p}}}
$$

Therefore, Theorem 3.2 reduces to the following statement.

Corollary 3.8. In the setting of Theorem 3.2, suppose that $\psi(N) \subset \mathfrak{k}$, and that $\mathrm{G} / \mathrm{K}$ is even-dimensional and equivariantly Spin. Then under the identification $\mathrm{KK}\left(\mathrm{C}_{0}(\mathrm{G} / \mathrm{K}) \rtimes \mathrm{G}, \mathbb{C}\right)=\widehat{\mathrm{R}}(\mathrm{K})$ by Morita equivalence, we have

$$
\operatorname{index}_{G}(\varepsilon, \psi)=\left(\left[\operatorname{ker}_{L^{2}}\left(D_{f \psi}^{\varepsilon_{N}}\right)^{+}\right]-\left[\operatorname{ker}_{L^{2}}\left(D_{f \psi}^{\varepsilon_{N}}\right)^{-}\right]\right) \otimes S_{\mathfrak{p}} \in \widehat{R}(K) .
$$

If $\mathrm{D}$ is a Spin ${ }^{\mathrm{c}}$-Dirac operator, we will see in Subsection 4.4 that the Gindex of its deformation satisfies the quantisation commutes with reduction principle in the setting of this subsection.

Remark 3.9. If $\mathrm{G}$ is semisimple, $\mathrm{G} / \mathrm{K}$ is even-dimensional, and $\operatorname{rank}(\mathrm{G}) \neq$ $\operatorname{rank}(K)$, then the element $\left[S_{\mathfrak{p}}\right] \in R(K)$ is zero, see (1.2.5) in [5]. (The arguments there actually imply that the same is true for reductive groups.) In this case, Corollary 3.8 is a vanishing result for the G-index, under the condition that $\psi(N) \subset \mathfrak{k}$. This is an exceptional situation, however, which shows how restrictive this condition is. In Section 4 , we will see many examples of nonzero G-indices. 
We have seen that the situations where the G-index of deformed Dirac operators has the potential to yield most information not accessible via existing index theory are those where

1. G is noncompact;

2. $M / G$ is noncompact; and

3. $\psi(N)$ is not contained in $\mathfrak{k}$ (for any choice of $p: M \rightarrow G / K$ ).

In Subsection 4.3, we will see a natural class of examples in this new setting.

In the rest of this section, we prove Proposition 3.1 and Theorem 3.2 .

\subsection{An explicit form of the Morita equivalence isomorphism}

Let us define the module $\mathcal{M}$, that implements the Morita equivalence $\mathrm{C}_{0}(\mathrm{G} / \mathrm{K}) \rtimes$ $\mathrm{G} \sim \mathrm{C}^{*} \mathrm{~K}$, as in Situation 10 in [32]. As a Hilbert $\mathrm{C}^{*} \mathrm{~K}$-module, it is the completion of $\mathrm{C}_{\mathrm{c}}(\mathrm{G})$ in the $\mathrm{C}^{*} \mathrm{~K}$-valued inner product given by

$$
\left(f, f^{\prime}\right)_{C^{* K}}(k)=\int_{G} \overline{f\left(g^{-1}\right)} f^{\prime}\left(g^{-1} k\right) d g
$$

for $f, f^{\prime} \in C_{c}(G)$ and $k \in K$. The right action by $C^{*} K$ is given by

$$
(f \psi)(g)=\int_{K} f(k g) \psi(k) d k,
$$

for $f \in C_{c}(G), \psi \in C(K)$ and $g \in G$. The representation $\pi_{\mathcal{M}}$ is given by

$$
\left(\pi_{\mathcal{M}}(\varphi) f\right)(g)=\int_{G} \varphi\left(g^{\prime}, g K\right) f\left(g^{\prime-1} g\right) \delta_{G}\left(g^{\prime}\right)^{1 / 2} d g^{\prime},
$$

for $\varphi \in C_{c}\left(G, C_{0}(G, K)\right), f \in C_{c}(G)$ and $g \in G$. (We will always identify maps from $G$ to $C_{0}(G / K)$ with functions on $G \times G / K$.)

Consider the class

$$
[\mathcal{M}]:=\left[\mathcal{M}, 0, \pi_{\mathcal{M}}\right] \in \mathrm{KK}\left(\mathrm{C}_{0}(\mathrm{G} / \mathrm{K}) \rtimes \mathrm{G}, \mathrm{C}^{*} \mathrm{~K}\right)
$$

defined by $\mathcal{M}$. Let $\mathcal{H}$ be a Hilbert space, and $\pi_{\mathrm{K}}: \mathrm{K} \rightarrow \mathrm{U}(\mathcal{H})$ a unitary representation. We will also use the symbol $\pi_{\mathrm{K}}$ for the corresponding representation

$$
\pi_{\mathrm{K}}: \mathrm{C}^{*} \mathrm{~K} \rightarrow \mathcal{B}(\mathcal{H}) .
$$

Let $\mathrm{F}$ be a K-equivariant, bounded operator on $\mathcal{H}$. 
Consider the representation

$$
\pi_{\mathrm{C}_{0}(\mathrm{G} / \mathrm{K}) \rtimes \mathrm{G}}: \mathrm{C}_{0}(\mathrm{G} / \mathrm{K}) \rtimes \mathrm{G} \rightarrow \mathcal{B}\left(\left(\mathrm{L}^{2}(\mathrm{G}) \otimes \mathcal{H}\right)^{\mathrm{K}}\right),
$$

defined by

$$
\left(\pi_{\mathrm{C}_{0}(\mathrm{G} / \mathrm{K}) \rtimes \mathrm{G}}(\varphi) \sigma\right)(\mathrm{g})=\int_{\mathrm{G}} \varphi\left(\mathrm{g}, \mathrm{g}^{\prime} \mathrm{K}\right) \delta_{\mathrm{G}}\left(\mathrm{g}^{\prime}\right)^{1 / 2} \sigma\left(\mathrm{g}^{\prime-1} \mathrm{~g}\right) \mathrm{dg}^{\prime}
$$

for $\varphi \in \mathrm{C}_{\mathrm{c}}\left(\mathrm{G}, \mathrm{C}_{0}(\mathrm{G} / \mathrm{K})\right), \sigma \in\left(\mathrm{L}^{2}(\mathrm{G}) \otimes \mathcal{H}\right)^{\mathrm{K}}$, and $\mathrm{g} \in \mathrm{G}$. Here $\delta_{\mathrm{G}}$ is the modular function on $\mathrm{G}$.

Lemma 3.10. There is a unitary isomorphism

$$
\Psi: \mathcal{M} \otimes \mathrm{C}^{* K} \mathrm{H} \rightarrow\left(\mathrm{L}^{2}(\mathrm{G}) \otimes \mathcal{H}\right)^{\mathrm{K}}
$$

that intertwines the representation $\pi_{\mathrm{C}_{0}(\mathrm{G} / \mathrm{K}) \rtimes \mathrm{G}}$ and the representation

$$
\pi_{\mathcal{M}} \otimes 1_{\mathcal{H}}: C_{0}(G / K) \rtimes G \rightarrow \mathcal{B}\left(\mathcal{M} \otimes_{C^{* K}} \mathcal{H}\right),
$$

and satisfies

$$
\Psi \circ\left(1_{\mathcal{M}} \otimes F\right)=\left(1_{L^{2}(G)} \otimes F\right) \circ \Psi .
$$

Proof. Consider the map

$$
\Psi: \mathrm{C}_{\mathrm{c}}(\mathrm{G}) \otimes_{\mathbb{C}} \mathcal{H} \rightarrow\left(\mathrm{C}_{\mathrm{c}}(\mathrm{G}) \otimes \mathcal{H}\right)^{\mathrm{K}}
$$

given by averaging over $\mathrm{K}$ :

$$
\Psi(f \otimes \xi)(g)=\int_{K} f(g k) \pi_{K}(k) \xi d k,
$$

for $f \in \mathrm{C}_{\mathrm{c}}(\mathrm{G}), \xi \in \mathcal{H}$ and $\mathrm{g} \in \mathrm{G}$. One checks that for all $\psi \in \mathrm{C}(\mathrm{K}) \subset \mathrm{C}^{*} \mathrm{~K}$, and $f$ and $\xi$ as above,

$$
\Psi(f \psi \otimes \xi)=\Psi\left(f \otimes \pi_{K}(\psi) \xi\right) .
$$

Hence the map $\Psi$ descends to a map

$$
\mathrm{C}_{\mathrm{c}}(\mathrm{G}) \otimes_{\mathrm{C}(\mathrm{K})} \mathcal{H} \rightarrow\left(\mathrm{C}_{\mathrm{c}}(\mathrm{G}) \otimes \mathcal{H}\right)^{\mathrm{K}}
$$

still denoted by $\Psi$. This map has the desired properties. 
Next, suppose that $\mathcal{H}$ has a $\mathrm{K}$-invariant $\mathbb{Z}_{2}$-grading, $\mathrm{F}$ is odd and selfadjoint, and the triple $\left(\mathcal{H}, F, \pi_{K}\right)$ is a Kasparov $\left(C^{*} K, \mathbb{C}\right)$-cycle. Let

$$
[\mathrm{F}] \in \mathrm{KK}\left(\mathrm{C}^{*} \mathrm{~K}, \mathbb{C}\right)
$$

be its class.

Proposition 3.11. The triple

$$
\left(\left(\mathrm{L}^{2}(\mathrm{G}) \otimes \mathcal{H}\right)^{\mathrm{K}}, 1_{\mathrm{L}^{2}(\mathrm{G})} \otimes \mathrm{F}, \pi_{\mathrm{C}_{0}(\mathrm{G} / \mathrm{K}) \rtimes \mathrm{G}}\right)
$$

is a Kasparov $\left(\mathrm{C}_{0}(\mathrm{G} / \mathrm{K}) \rtimes \mathrm{G}, \mathbb{C}\right)$-cycle, and its class in $\mathrm{KK}\left(\mathrm{C}_{0}(\mathrm{G} / \mathrm{K}) \rtimes \mathrm{G}, \mathbb{C}\right)$ equals

$$
[\mathcal{M}] \otimes_{C^{* K}}[F] .
$$

Proof. Since the operator $\mathrm{F}$ commutes with the representation of $\mathrm{C}^{*} \mathrm{~K}$ in $\mathcal{H}$, the operator $1_{\mathcal{M}} \otimes \mathrm{F}$ on $\mathcal{M} \otimes{ }_{C^{*} K} \mathcal{H}$ is well-defined. Furthermore, the Kasparov product of $[\mathcal{M}]$ and $[\mathrm{F}]$ is represented by the Kasparov $\left(\mathrm{C}_{0}(\mathrm{G} / \mathrm{K}) \rtimes \mathrm{G}, \mathbb{C}\right)$ cycle

$$
\left(\mathcal{M} \otimes{ }_{\mathrm{C}^{*} \mathrm{~K}} \mathcal{H}, 1_{\mathcal{M}} \otimes \mathrm{F}, \pi_{\mathcal{M}} \otimes 1_{\mathcal{H}}\right) .
$$

(See e.g. [7], Example 18.3.2(a).) This Kasparov cycle is unitarily equivalent to 3.13 by Lemma 3.10 .

\subsection{Product metrics}

The K-invariant metric $B_{\mathfrak{p}}$ on $\left.\mathrm{TM}\right|_{\mathrm{N}}$ extends to a G-invariant Riemannian metric on TM, which we still denote by $B_{\mathfrak{p}}$. By Proposition 3.13 in [21], this Riemannian metric leads to the same G-index as the original metric, as long as $M$ is complete with respect to $B_{\mathfrak{p}}$.

Lemma 3.12. The manifold $M$ is complete in the metric $B_{\mathfrak{p}}$.

Proof. Note that $\mathrm{G}$ is complete in the left invariant Riemannian metric $\mathrm{B}_{\mathrm{G}}$ defined by the inner product on $\mathfrak{g}$ used in the definition of $B_{\mathfrak{p}}$. Since $M$ is complete in $B$, the slice $N$ is complete in the metric $\left.B\right|_{T N}$. Hence $G \times N$ is complete in the product metric $B_{G} \times\left. B\right|_{T N}$. The quotient $M$ of $G \times N$ is therefore complete in the distance function induced by the Riemannian distance on $G \times N$ (see Proposition 3.1 in [2]). This equals the distance function defined by the Riemannian metric $B_{\mathfrak{p}}$, so the claim follows.

Let $\mathrm{L}^{2}\left(\mathcal{E}, \mathrm{B}_{\mathfrak{p}}\right)$ be the $\mathrm{L}^{2}$-space of sections of $\mathcal{E}$, defined with respect to the Riemannian density associated to $B_{\mathfrak{p}}$. We use the metric $B_{\mathfrak{p}}$ for two reasons. 
The first is that Lemma 3.5 is true for this metric. The second is that it allows us to decompose the space $\mathrm{L}^{2}\left(\mathcal{E}, \mathrm{B}_{\mathfrak{p}}\right)$ in a way that will allow us to apply Proposition 3.11 .

For this decomposition, we consider the map

$$
\Phi:\left(\mathrm{C}_{\mathrm{c}}(\mathrm{G}) \otimes \Gamma_{\mathrm{c}}\left(\left.\varepsilon\right|_{\mathrm{N}}\right)\right)^{\mathrm{K}} \rightarrow \Gamma_{\mathrm{c}}(\varepsilon)
$$

given by

$$
\Phi(\varphi \otimes s)(g n)=\varphi(g) g \cdot s(n),
$$

for $\varphi \in \mathrm{C}_{\mathrm{c}}(\mathrm{G})$ and $s \in \Gamma_{\mathrm{c}}\left(\left.\mathcal{E}\right|_{\mathrm{N}}\right)$ such that $\varphi \otimes s$ is $\mathrm{K}$-invariant, and $\mathrm{g} \in \mathrm{G}$ and $n \in N$. In general the K-invariant simple tensors of the form $\varphi \otimes s$ may not span the whole space $\left(\mathrm{C}_{\mathrm{c}}(\mathrm{G}) \otimes \Gamma_{\mathrm{c}}\left(\left.\varepsilon\right|_{N}\right)\right)^{\mathrm{K}}$. Then we extend $\Psi$ linearly to sums of tensors that are K-invariant, while their individual terms may not be. We will tacitly use this convention in the rest of this section.

Lemma 3.13. The map $\Phi$ extends to a G-equivariant, unitary isomorphism

$$
\Phi:\left(\mathrm{L}^{2}(\mathrm{G}) \otimes \mathrm{L}^{2}\left(\left.\varepsilon\right|_{\mathrm{N}}\right)\right)^{\mathrm{K}} \rightarrow \mathrm{L}^{2}\left(\mathcal{E}, \mathrm{B}_{\mathfrak{p}}\right) .
$$

Proof. Equivariance of $\Phi$ follows directly from the definitions. For surjectivity, note that $\Phi\left(\left(\mathrm{C}_{\mathfrak{c}}(\mathrm{G}) \otimes \Gamma_{\mathrm{c}}\left(\left.\mathcal{E}\right|_{\mathrm{N}}\right)\right)^{\mathrm{K}}\right)$ is dense in $\mathrm{L}^{2}\left(\varepsilon, \mathrm{B}_{\mathfrak{p}}\right)$. To show that $\Phi$ is an isometry, we consider the G-invariant measure $d[g, n]$ on $M=G \times{ }_{K} N$ induced by the Riemannian density on $\mathrm{G} \times \mathrm{N}$ associated to the product metric $B_{G} \times\left. B\right|_{T N}$ used in the proof of Lemma 3.12. (See e.g. [8], Chapter VII, Section 2.2, Proposition 4b.) By a direct verification, the map $\Phi$ is unitary with respect to that measure. One can show that the measure $d[g, n]$ equals the one given by the Riemannian density associated to $B_{\mathfrak{p}}$. (See Lemma 5.1 in [17].)

\subsection{Morita equivalence and the G-index}

The comments on the Riemannian metric $B_{\mathfrak{p}}$ in Subsection 3.5 allow us to deduce Theorem 3.2 from Proposition 3.11.

For any K-equivariant (real or complex) vector bundle $\mathrm{E} \rightarrow \mathrm{N}$, consider the G-equivariant vector bundle

$$
\mathrm{G} \times \times_{\mathrm{K}} \mathrm{E} \rightarrow \mathrm{M}
$$

Analogously to 3.14, we have G-equivariant map

$$
\Phi_{\mathrm{E}}:\left(\mathrm{C}^{\infty}(\mathrm{G}) \otimes \Gamma^{\infty}(\mathrm{E})\right)^{\mathrm{K}} \rightarrow \Gamma^{\infty}\left(\mathrm{G} \times{ }_{\mathrm{K}} \mathrm{E}\right),
$$


given by

$$
\Phi_{\mathrm{E}}(\varphi \otimes s)(\mathrm{gn})=\varphi(\mathrm{g}) \mathrm{g} \cdot \mathrm{s}(\mathrm{n}),
$$

for $\varphi \in \mathrm{C}^{\infty}(\mathrm{G})$ and $s \in \Gamma^{\infty}(\mathrm{E})$ such that $\varphi \otimes s$ is $\mathrm{K}$-invariant, and $\mathrm{g} \in \mathrm{G}$ and $n \in N$. If $E=\left.\mathcal{E}\right|_{N}$, this gives

$$
\Phi:=\Phi_{\left.\mathcal{E}\right|_{N}}:\left(C^{\infty}(G) \otimes \Gamma^{\infty}\left(\left.\mathcal{E}\right|_{N}\right)\right)^{K} \rightarrow \Gamma^{\infty}(\mathcal{E}) .
$$

Recall that we used the restricted connection $\nabla^{\left.\mathcal{E}\right|_{N}}$ to define the Dirac operator $\mathrm{D}^{\mathrm{N}}$ in (3.1). We will also use a decomposition of the Dirac operator D. To define this decomposition, we recall that we have

$$
\mathrm{M} \cong \mathrm{G} \times \times_{\mathrm{K}} \mathrm{N}
$$

as in (2.1). We have a G-equivariant isomorphism of vector bundles

$$
\mathrm{TM} \cong \mathrm{p}^{*} \mathrm{~T}(\mathrm{G} / \mathrm{K}) \oplus \mathrm{G} \times_{\mathrm{K}} \mathrm{TN} .
$$

This decomposition of TM yields two projections

$$
\begin{gathered}
\mathrm{p}_{\mathrm{G} / \mathrm{K}}: \mathrm{TM} \rightarrow \mathrm{p}^{*} \mathrm{~T}(\mathrm{G} / \mathrm{K}) ; \\
\mathrm{p}_{\mathrm{N}}: \mathrm{TM} \rightarrow \mathrm{G} \times_{\mathrm{K}} \mathrm{TN} .
\end{gathered}
$$

Identifying $T^{*} M \cong T M$ via the Riemannian metric as before, we obtain two partial Dirac operators

$$
\begin{gathered}
\mathrm{D}_{\mathrm{G} / \mathrm{K}}: \Gamma^{\infty}(\mathcal{E}) \stackrel{\nabla^{\varepsilon}}{\longrightarrow} \Gamma^{\infty}(\mathrm{TM} \otimes \mathcal{E}) \stackrel{\mathrm{p}_{\mathrm{G} / \mathrm{K}} \otimes 1_{\varepsilon}}{\longrightarrow} \Gamma^{\infty}\left(\mathrm{p}^{*} \mathrm{~T}(\mathrm{G} / \mathrm{K}) \otimes \mathcal{E}\right) \stackrel{\mathrm{c}}{\rightarrow} \Gamma^{\infty}(\mathcal{E}) ; \\
\mathrm{D}_{\mathrm{N}}: \Gamma^{\infty}(\varepsilon) \stackrel{\nabla^{\varepsilon}}{\longrightarrow} \Gamma^{\infty}(\mathrm{TM} \otimes \mathcal{E}) \stackrel{\mathrm{p}_{\mathrm{N}} \otimes 1_{\varepsilon}}{\longrightarrow} \Gamma^{\infty}(\mathrm{G} \times \mathrm{K} \mathrm{TN} \otimes \mathcal{E}) \stackrel{\mathrm{c}}{\rightarrow} \Gamma^{\infty}(\mathcal{\varepsilon}) .
\end{gathered}
$$

Since $p_{G / K}+p_{N}$ is the identity map on TM, we have

$$
\mathrm{D}=\mathrm{D}_{\mathrm{G} / \mathrm{K}}+\mathrm{D}_{\mathrm{N}} \text {. }
$$

This decomposition played a crucial role in the etimates in [21].

The proofs of the following two lemmas are straightforward.

Lemma 3.14. The following diagram commutes:

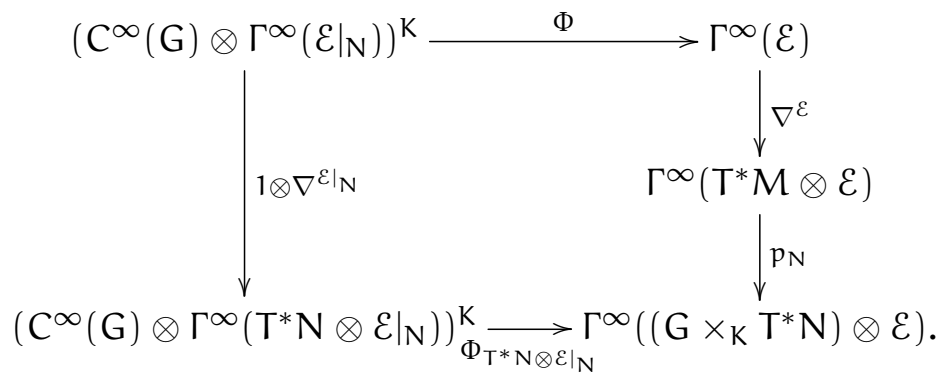


Lemma 3.15. One has

$$
\Phi \circ\left(1 \otimes \mathrm{D}^{\mathrm{N}}\right)=\mathrm{D}_{\mathrm{N}} \circ \Phi
$$

Let

$$
c_{\mathfrak{p}}:\left(T M, B_{\mathfrak{p}}\right) \rightarrow \operatorname{End}(\mathcal{E})
$$

be the G-equivariant extension of (3.4). Let $f \in C^{\infty}(M)^{G}$. Then

$$
\Phi \circ\left(1 \otimes c_{\mathfrak{p}}\left(\left.f v^{\psi}\right|_{N}\right)\right)=c_{\mathfrak{p}}\left(f v^{\psi}\right) \circ \Phi .
$$

So Lemma 3.15 implies that

$$
\Phi \circ\left(1 \otimes D_{\left.f \psi\right|_{N}}^{N}\right)=\left(D_{N}-\sqrt{-1} c_{p}\left(f v^{\psi}\right)\right) \circ \Phi .
$$

This allows us to prove Theorem 3.2

Proof of Theorem 3.2. Let $\rho \in \mathrm{C}^{\infty}(M)^{\mathrm{G}}$ be as in Theorem 2.1, for the Riemannian metric $B_{\mathfrak{p}}$ and the Clifford action $c_{\mathfrak{p}}$. Let $\rho_{N} \in C^{\infty}(N)^{K}=C^{\infty}(M)^{G}$ be as in Proposition 3.1. Suppose $f \in C^{\infty}(M)$ is $\max \left(\rho, \rho_{N}\right)$-admissible. For $t \in \mathbb{R}$, consider the operator

$$
D_{f \psi, t}:=D_{N}+t D_{G / K}-\sqrt{-1} f c_{p}\left(v^{\psi}\right) .
$$

Here $D_{N}$ and $D_{G / K}$ are defined with respect to $B_{\mathfrak{p}}$ and $c_{\mathfrak{p}}$. The arguments in Sections 4 and 5 of [21], with $D_{G / K}$ replaced by $t D_{G / K}$, show that for all $t>0$, the operator $D_{f \psi, t}$ is G-Fredholm for $p$. For $t=0$, this operator is not elliptic. So we cannot apply the Rellich lemma as in Subsection3.5 of [21] to show that $D_{f \psi, 0}$ is G-Fredholm for $p$. However, we saw in Proposition 3.1 that the operator $D_{f \psi}^{N}$ is K-Fredholm. Proposition 3.11 therefore implies that

$$
\left(\left(\mathrm{L}^{2}(\mathrm{G}) \otimes \mathrm{L}^{2}\left(\left.\mathcal{E}\right|_{\mathrm{N}}\right)\right)^{\mathrm{K}}, 1_{\mathrm{L}^{2}(\mathrm{G})} \otimes \frac{\mathrm{D}_{\mathrm{f} \psi}^{\mathrm{N}}}{\sqrt{\left(\mathrm{D}_{\mathrm{f} \psi}^{\mathrm{N}}\right)^{2}+1}}, \pi_{\mathrm{C}_{0}(\mathrm{G} / \mathrm{K}) \rtimes \mathrm{G}}\right)
$$

is a Kasparov $\left(\mathrm{C}_{0}(\mathrm{G} / \mathrm{K}) \rtimes \mathrm{G}, \mathbb{C}\right)$-cycle. The isomorphism $\Phi$ intertwines the representations $\pi_{\mathrm{C}_{\mathrm{O}}(\mathrm{G} / \mathrm{K}) \rtimes \mathrm{G}}$ and $\pi_{\mathrm{G}, \mathrm{G} / \mathrm{K}}$, up to the factor $\delta_{\mathrm{G}}\left(\mathrm{g}^{\prime}\right)^{1 / 2}$ in the definition (3.12) of $\pi_{\mathrm{C}_{0}(\mathrm{G} / \mathrm{K}) \rtimes}$. On pages 131 and 132 of [34], it is explained how to remove this factor. Then Lemma 3.13 and the equality (3.19) imply that (3.20) is unitarily equivalent to

$$
\left(\mathrm{L}^{2}(\varepsilon), \frac{\mathrm{D}_{\mathrm{f} \psi, 0}}{\sqrt{\left(\mathrm{D}_{\mathrm{f} \psi, 0}\right)^{2}+1}}, \pi_{\mathrm{G}, \mathrm{G} / \mathrm{K}}\right)
$$


The triple (3.21) is therefore also a Kasparov $\left(\mathrm{C}_{0}(\mathrm{G} / \mathrm{K}) \rtimes \mathrm{G}, \mathbb{C}\right)$-cycle, which is to say that $D_{f \psi, 0}$ is G-Fredholm for $p$.

We conclude that for all $t \geq 0$, the operator $D_{f \psi, t}$ is G-Fredholm for $p$. So using an operator homotopy, we obtain

$$
\operatorname{index}_{G}^{p} D_{f \psi, 0}=\operatorname{index}_{G}^{p} D_{f \psi, 1} \quad \in K K\left(C_{0}(G / K) \rtimes G, \mathbb{C}\right) .
$$

By Proposition 3.13 in [21], we have

$$
\operatorname{index}_{G}(\mathcal{E}, \psi)=\operatorname{index}_{G}^{p} D_{f \psi, 1} \text {. }
$$

Since the triples (3.20) and (3.21) are unitarily equivalent, Proposition 3.11 implies that

$$
\operatorname{index}_{G}^{p} D_{f \psi, 0}=[\mathcal{M}] \otimes_{C^{*} K} \operatorname{index}_{K}\left(D_{\left.f \psi\right|_{N}}^{N}\right) \text {. }
$$

The result now follows from Lemma 2.9 in [21].

\section{Properties of the G-index}

The G-index of deformed Dirac operators turns out to have several interesting properties. We already saw that it equals Braverman's index if $\mathrm{G}$ is compact (see Lemma Lemma 2.9 in [21]). If $M / G$ is compact, we describe how it is related to the analytic assembly map and to an index used by Mathai and Zhang. We work out examples where $M=T^{*}(G / K)$ and where $G=\mathbb{R}$. The first of these examples gives rise to a K-homological version of the Dirac induction isomorphism described in Subsection 2.3. Finally, we show that for $\operatorname{Spin}^{\mathrm{c}}$-Dirac operators, the index satisfies the quantisation commutes with reduction principle that was originally formulated in symplectic geometry.

\subsection{The analytic assembly map and the Mathai-Zhang index}

In this subsection, we suppose that $M / G$ is compact, but $M$ and $G$ may be noncompact. Then the G-index is closely related to the analytic assembly map and an index defined by Mathai and Zhang [28].

Let

- $\mathrm{C}^{*} \mathrm{G}$ now denote the maximal group $\mathrm{C}^{*}$-algebra of $\mathrm{G}$;

- $\mu_{M}^{G}: K^{G}\left(C_{0}(M), \mathbb{C}\right) \rightarrow K K\left(\mathbb{C}, C^{*} G\right)$ be the analytic assembly map (2.6); 
- $\left[1_{K}\right] \in \mathrm{KK}\left(\mathbb{C}, \mathrm{C}_{0}(\mathrm{G} / \mathrm{K}) \rtimes \mathrm{G}\right) \cong \mathrm{R}(\mathrm{K})$ be the class corresponding to the trivial representation of $\mathrm{K}$;

- $\left[1_{\mathrm{G}}\right] \in \mathrm{KK}\left(\mathrm{C}^{*} \mathrm{G}, \mathbb{C}\right)$ be the class corresponding to the trivial representation of $\mathrm{G}$, equal to the class of the $*$-homomorphism $\mathrm{I}^{\mathrm{G}}: \mathrm{C}^{*} \mathrm{G} \rightarrow \mathbb{C}$ given on $L^{1}(G)$ by integrating functions over $G$.

The Mathai-Zhang index was defined in Definition 2.4 in [28], for Dirac operators. It is a numerical index, which is defined in terms of the G-invariant part of the kernel of an operator.

As before, let $p: M \rightarrow G / K$ be a smooth, equivariant map. Since $M / G$ is compact, the map $p$ is proper. So it induces $p^{*}: C_{0}(G / K) \rightarrow C_{0}(M)$, and hence $\left(p_{G}\right)^{*}: C_{0}(G / K) \rtimes G \rightarrow C_{0}(M) \rtimes G$. Let

$$
j^{G}: K^{G}\left(C_{0}(M), \mathbb{C}\right) \rightarrow K K\left(C_{0}(M) \rtimes G, C^{*} G\right)
$$

be the descent map ([23], Section 3.11).

Proposition 4.1. If $\mathrm{M} / \mathrm{G}$ is compact, then there are maps index $_{\mathrm{G}}$ and index $\mathrm{x}_{\mathrm{MZ}}$, which on K-homology classes defined by elliptic operators are given by the G-index and the Mathai-Zhang index of these operators, respectively, such that the following diagram commutes:

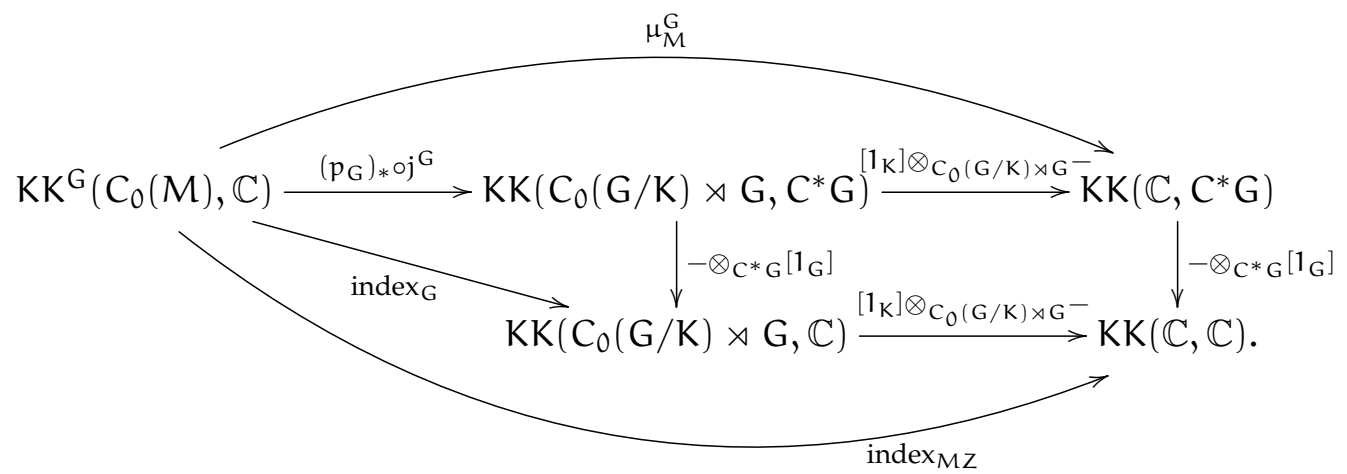

This proposition implies that the assembly map and the G-index of an elliptic operator D can both be recovered from the class

$$
\left(p_{G}\right)_{*} \circ j^{G}[D] \in K K\left(C_{0}(G / K) \rtimes G, C^{*} G\right) .
$$

Furthermore, the Mathai-Zhang index can be recovered from either of these two indices, via the Kasparov product with $\left[1_{\mathrm{G}}\right]$ and $\left[1_{\mathrm{K}}\right]$, respectively. 
To prove Proposition 4.1, we consider an odd, self-adjoint, elliptic, Gequivariant differential operator on $\mathcal{E} \rightarrow M$. Then we have the class

$$
[\mathrm{D}]_{\mathrm{C}_{0}(M) \rtimes \mathrm{G}}=\left[\mathrm{L}^{2}(\mathcal{E}), \frac{\mathrm{D}}{\sqrt{\mathrm{D}^{2}+1}}, \pi_{\mathrm{G}, \mathrm{C}_{0}(M)}\right] \in \mathrm{KK}\left(\mathrm{C}_{0}(\mathrm{M}) \rtimes \mathrm{G}, \mathbb{C}\right)
$$

as in (the elliptic case of) Proposition 6.4 in [24]. Here $\pi_{G, C_{0}(M)}: C_{0}(M) \rtimes$ $\mathrm{G} \rightarrow \mathcal{B}\left(\mathrm{L}^{2}(\mathcal{E})\right)$ is induced by the representations of $\mathrm{C}_{0}(M)$ and $\mathrm{G}$ in $\mathrm{L}^{2}(\mathcal{E})$.

Consider the class $[D] \in \mathrm{KK}^{\mathrm{G}}\left(\mathrm{C}_{0}(M), \mathbb{C}\right)$ defined by $\mathrm{D}$.

Lemma 4.2. We have

$$
[D]_{C_{0}(M) \rtimes G}=j^{G}[D] \otimes C^{* G}\left[1_{G}\right] .
$$

Proof. One can check that

$$
j^{G}[D]=\left[L^{2}(\mathcal{E}) \otimes C^{*} G, \frac{D_{f \psi}}{\sqrt{D_{f \psi}^{2}+1}} \otimes 1, \pi\right] \in K K\left(C_{0}(M) \rtimes G, C^{*} G\right),
$$

where $\pi: C_{0}(M) \rtimes G \rightarrow \mathcal{B}\left(L^{2}(\varepsilon) \otimes C^{*} G\right)$ is given by

$$
\pi(\varphi \otimes f)(s \otimes \psi)(g)=\int_{G} \varphi\left(g^{\prime}\right) \psi\left(g^{\prime-1} g\right) f^{\prime} \cdot s d^{\prime},
$$

for $\varphi, \psi \in \mathrm{C}_{\mathrm{c}}(\mathrm{G}), f \in \mathrm{C}_{0}(\mathrm{M}), \mathrm{s} \in \mathrm{L}^{2}(\varepsilon)$ and $\mathrm{g} \in \mathrm{G}$.

The product with $\left[1_{\mathrm{G}}\right]$ is just the map functorially induced by the map $\mathrm{I}^{\mathrm{G}}$. So taking this product, we obtain

$$
j^{G}[D] \otimes C^{*} G\left[1_{G}\right]=\left[L^{2}(\mathcal{E}) \otimes C^{*} G \otimes_{I_{G}} \mathbb{C}, \frac{D_{f \psi}}{\sqrt{D_{f \psi}^{2}+1}} \otimes 1 \otimes 1, \pi \otimes 1\right] .
$$

Now we have the unitary isomorphism

$$
\Phi: \mathrm{L}^{2}(\mathcal{E}) \otimes \mathrm{C}^{*} \mathrm{G} \otimes_{\mathrm{I}_{\mathrm{G}}} \mathbb{C} \stackrel{\cong}{\cong} \mathrm{L}^{2}(\mathcal{E})
$$

given by

$$
\Phi(\mathrm{s} \otimes \psi \otimes z)=z \mathrm{I}^{\mathrm{G}}(\psi) \mathrm{s},
$$

for $s \in \mathrm{L}^{2}(\mathcal{E}), \psi \in \mathrm{C}_{\mathrm{c}}(\mathrm{G})$ and $z \in \mathbb{C}$. By a direct computation, we find that for all $\varphi, \psi \in \mathrm{C}_{\mathrm{c}}(\mathrm{G}), \mathrm{f} \in \mathrm{C}_{0}(\mathrm{M}), \mathrm{s} \in \mathrm{L}^{2}(\mathcal{E})$ and $z \in \mathbb{C}$,

$$
\Phi \circ(\pi \otimes 1)(\varphi \otimes f)(s \otimes \psi \otimes z)=\pi_{G, M}(\varphi \otimes f) \Phi(s \otimes \psi \otimes z) .
$$


Remark 4.3. Proposition 6.4 in [24] states that the class $[D]_{C_{0}(M) \rtimes G}$ is welldefined even if $D$ is just transversally elliptic. If $D$ is elliptic, then it also defines a class in $\mathrm{K}^{\mathrm{G}}\left(\mathrm{C}_{0}(M), \mathbb{C}\right)$, and Lemma 4.2 is a relation between these classes.

Define the map index ${ }_{G}$ by commutativity of the following diagram:

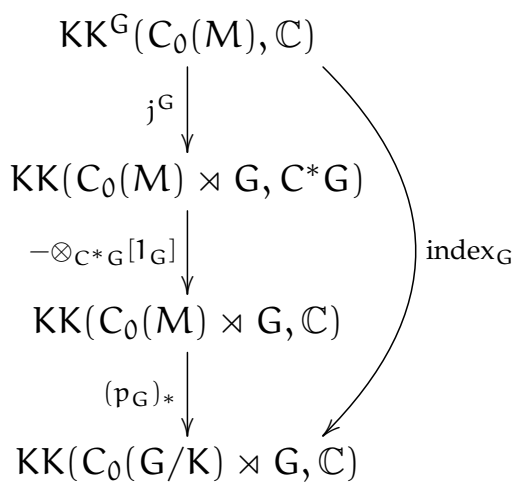

Then Lemma 4.2 shows that this map indeed equals the G-index on classes defined by elliptic operators.

Define the map

$$
\operatorname{index}_{\mathrm{MZ}}: \mathrm{KK}^{\mathrm{G}}\left(\mathrm{C}_{0}(\mathrm{M}), \mathbb{C}\right) \rightarrow \mathrm{KK}(\mathbb{C}, \mathbb{C})=\mathbb{Z}
$$

as the composition of the analytic assembly map and the Kasparov product over $C^{*} G$ with $\left[1_{G}\right]$. Bunke showed in the appendix to [28] that this corresponds to the Mathai-Zhang index on classes of Dirac operators.

Proof of Proposition 4.1. Consider the diagram

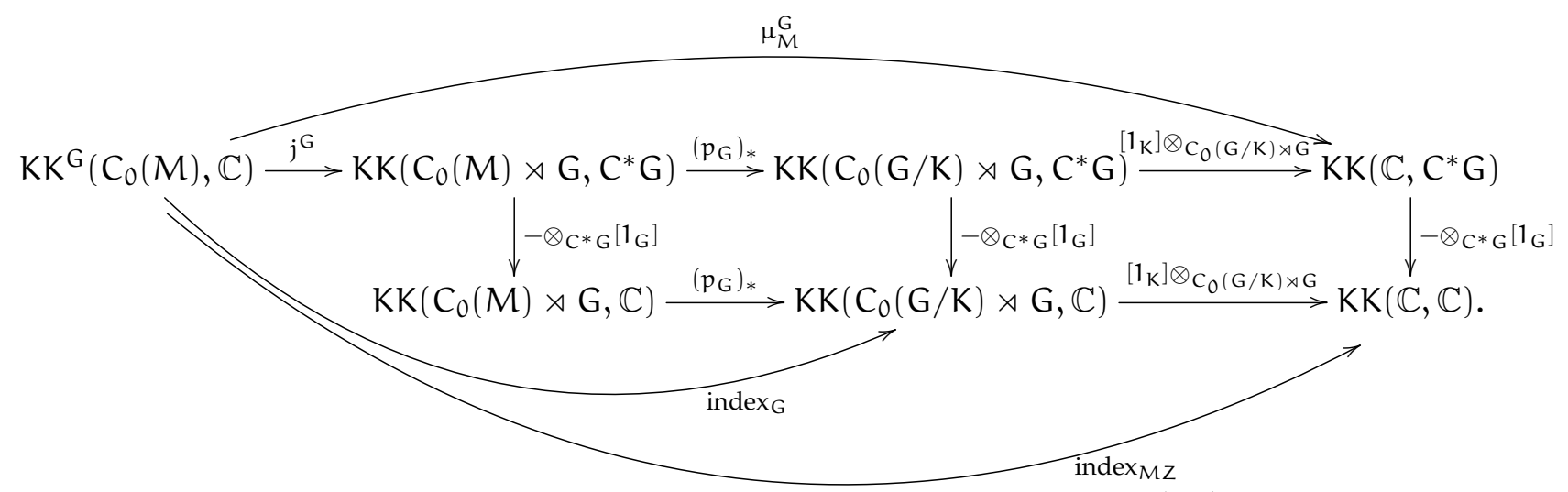


The two squares with the map $\left(\mathrm{p}_{\mathrm{G}}\right)_{*}$ and the products with $\left[1_{\mathrm{K}}\right]$ and $\left[1_{\mathrm{G}}\right]$, commute by the basic functoriality and associativity properties of KK-theory. The class $\left[1_{K}\right] \in K K\left(\mathbb{C}, C_{0}(G / K) \rtimes G\right)$ equals the class defined by a cutoff function on $\mathrm{G} / \mathrm{K}$. Hence commutativity of the top part of this diagram is one of the standard definitions of the assembly map, combined with the fact that the assembly map behaves naturally with respect to the map $p_{*}$. The remaining parts of the diagram commute by the comments made above. We conclude that the whole diagram (4.3) commutes.

Remark 4.4. In [10, 18], a generalisation of the Mathai-Zhang index of deformed Dirac operators to non-cocompact actions is studied. If $G$ is compact, then

$$
\left.\left[1_{K}\right] \otimes C_{0}(G / K) \rtimes G \text { index } \operatorname{dD}_{f \psi}\right)
$$

is the index studied in [10, 18]. Since we saw in Proposition 4.1 that this is also true if $M / G$, instead of $G$, is compact, this leads the authors to suspect that it holds in general.

Example 4.5. If $\mathrm{G}=\mathrm{K}$ is compact, so that $\mathrm{M}$ is as well, then the class 4.2 in

$$
\mathrm{KK}\left(\mathrm{C}^{*} \mathrm{~K}, \mathrm{C}^{*} \mathrm{~K}\right)=\operatorname{Hom}(\mathrm{R}(\mathrm{K}), \mathrm{R}(\mathrm{K}))
$$

is taking the tensor product with the usual equivariant index of D. The map

$$
\mathrm{I}_{*}^{\mathrm{K}}: \operatorname{Hom}(\mathrm{R}(\mathrm{K}), \mathrm{R}(\mathrm{K})) \rightarrow \mathrm{R}(\mathrm{K})
$$

is now given by applying operators on $R(K)$ to the trivial representation. As expected, applying this to the class (4.2) yields the equivariant index of $D$, i.e. its image under the assembly map for compact groups.

The Kasparov product with $\left[1_{\mathrm{K}}\right] \in \mathrm{KK}\left(\mathrm{C}^{*} \mathrm{~K}, \mathbb{C}\right)$ is the map

$$
\operatorname{Hom}(R(K), R(K)) \rightarrow \operatorname{Hom}(R(K), \mathbb{Z})
$$

given by taking the dimension of the invariant part after applying an operator on $R(K)$. Applying this map to (4.2) yields the map $R(K) \rightarrow \mathbb{Z}$ that maps the class of $V \in \hat{R}$ to

$$
\operatorname{dim}\left(\operatorname{index}_{K}(D) \otimes V\right)^{K}=\left[\operatorname{index}_{K}(D): V^{*}\right]=\left[\operatorname{index}_{K}(D): V\right]
$$

(Here index $\mathrm{K}_{\mathrm{K}}$ now denotes the usual equivariant index.) In other words, we recover the fact that the $\mathrm{K}$-index of $\mathrm{D}$ as defined in [21] is the image in $\widehat{R}(K)$ of the usual K-index of D. 


\subsection{Another relation with the assembly map}

As in Subsection 4.1. assume that $M / G$ is compact. In addition, we assume that $\mathrm{G} / \mathrm{K}$ is even-dimensional and equivariantly Spin. Consider the Dirac induction isomorphism

$$
\mathrm{D}-\operatorname{Ind}_{\mathrm{K}}^{\mathrm{G}}: \mathrm{R}(\mathrm{K}) \stackrel{\cong}{\mathrm{K} K}\left(\mathbb{C}, \mathrm{C}_{\mathrm{r}}^{*} \mathrm{G}\right)
$$

described in Subsection 2.3. We now use the analytic assembly map with respect to the reduced group $C^{*}$-algebra $C_{r}^{*} G$, which we still denote by $\mu_{M}^{G}$.

In this setting, we can express the G-index in terms of the assembly map.

Proposition 4.6. Under the identification $\mathrm{KK}\left(\mathrm{C}_{0}(\mathrm{G} / \mathrm{K}) \rtimes \mathrm{G}, \mathbb{C}\right)=\widehat{\mathrm{R}}(\mathrm{K})$ via Morita equivalence, we have

$$
\operatorname{index}_{G}\left(D_{M}\right)=\left(D-I n d_{K}^{G}\right)^{-1}\left(\mu_{M}^{G}\left[D_{M}\right]\right) \otimes S_{\mathfrak{p}} \quad \in R(K) \hookrightarrow \widehat{R}(K) .
$$

Proof. Since we may take $\psi=0$ in the cocompact case, the condition in Subsection 3.3 that $\psi(N) \subset \mathfrak{k}$ is automatically satisfied. By Corollary 3.8 . we therefore have

$$
\operatorname{index}_{G}\left(D_{M}\right)=[\mathcal{M}] \otimes_{C^{*} K}\left(\operatorname{index}_{K}\left(D^{\varepsilon_{N}}\right) \otimes S_{\mathfrak{p}}\right) .
$$

Hence the claim follows from the fact that

$$
\mu_{M}^{G}[D]=D-\operatorname{Ind}_{K}^{G}\left(\operatorname{index}_{K}\left(D^{\varepsilon_{N}}\right)\right) .
$$

This was proved for $\operatorname{Spin}^{\mathrm{c}}$-Dirac operators in Theorem 4.5 in [16] and Theorem 4.8 in [19]. The arguments apply to general Dirac operators, however.

Proposition 4.6 implies that the G-index of Dirac operators is determined by the assembly map. Conversely, the index $\mu_{M}^{G}\left[D_{M}\right]$ can be expressed in terms of index ${ }_{G}\left(D_{M}\right)$ precisely if tensoring with $S_{\mathfrak{p}}$ is an injective operation on $R(K)$.

Lemma 4.7. Suppose that $\mathrm{G}$ is semisimple with discrete series, i.e. $\operatorname{rank}(\mathrm{G})=$ $\operatorname{rank}(\mathrm{K})$. Then the map from $\mathrm{R}(\mathrm{K})$ to $\mathrm{R}(\mathrm{K})$ given by the tensor product with $\mathrm{S}_{\mathfrak{p}}$ is injective. 
Proof. Let $\mathrm{T}^{\mathrm{reg}}$ be the set of regular elements in a maximal torus $\mathrm{T}$ of $\mathrm{K}$. Since $\operatorname{rank}(\mathrm{G})=\operatorname{rank}(\mathrm{K})$, it was noted in Remark 2.2 in [31] that the character $\chi_{S_{\mathfrak{p}}}=\chi_{S_{\mathfrak{p}}^{-}}-\chi_{S_{\mathfrak{p}}^{-}}$of $S_{\mathfrak{p}}$ satisfies

$$
\left.\chi_{s_{p}}\right|_{T}=\prod_{\alpha \in R_{n}^{+}}\left(e^{\alpha / 2}-e^{-\alpha / 2}\right),
$$

where $\alpha$ runs over a set of positive noncompact roots. This function is nonzero on $T^{\text {reg }}$, and hence on the open dense subset $K \cdot T^{\text {reg }} \subset K$, where $\mathrm{K}$ acts on itself by conjugation. Therefore, multiplication by $\chi_{S_{p}}$ is injective.

In the setting of this lemma, we have

$$
\mu_{M}^{G}\left[D_{M}\right]=D-\operatorname{Ind}_{K}^{G}[V],
$$

where the character of $[V] \in R(K)$ equals the character of index $\operatorname{lex}_{G}\left(D_{M}\right)$ divided by $\chi_{s_{p}}$.

Example 4.8. Suppose that $M=G / K$. For $V \in \hat{R}$, let $D_{V}$ be the Dirac operator on the Clifford module $G \times_{K}\left(S_{\mathfrak{p}} \otimes V\right) \rightarrow G / K$, as defined in (2.4). Since $M / G$ is compact, this operator is G-Fredholm without the need of a deformation term. Since $M$ is now a homogeneous space, all operators on G-equivariant vector bundles over $M$ are transversally elliptic, including the zero operator. Therefore, Proposition 6.4 in [24] implies that

$$
\operatorname{index}_{G}\left(D_{V}\right)=\left[\left(L^{2}(G) \otimes S_{\mathfrak{p}} \otimes V\right)^{K}, 0, \pi_{G, G / K}\right] \quad \in K K\left(C_{0}(G / K) \rtimes G, \mathbb{C}\right),
$$

via a linear operator homotopy. By Proposition 2.10 in [21], this class corresponds to $S_{\mathfrak{p}} \otimes V \in \widehat{R}(K)$. The right hand side of (4.4) for this operator equals $V \otimes S_{\mathfrak{p}}$, so that we obtain an independent verification of Proposition 4.6 in this case.

One could view the G-index of $D_{V}$ as a K-homological analogue of the Dirac induction of $V$. The twist by $S_{\mathfrak{p}}$ one obtains in this way makes this slightly unnatural though, also in view of Remark 3.9. In Subsection 4.3. we define a more natural notion of K-homological Dirac induction, using a non-cocompact action.

\subsection{K-homological Dirac induction}

We assume that $G / K$ is even-dimensional and equivariantly Spin. The Dirac induction isomorphism

$$
\mathrm{D}-\operatorname{Ind}_{\mathrm{K}}^{\mathrm{G}}: \mathrm{R}(\mathrm{K}) \rightarrow \mathrm{K}_{*}\left(\mathrm{C}_{\mathrm{r}}^{*} \mathrm{G}\right)
$$


involves Dirac operators on G/K. Using the G-index, we define a version of Dirac induction in terms of Dirac operators on the non-cocompact manifold

$$
M=T^{*}(G / K) \cong G \times K \mathfrak{p} .
$$

(We identify $\mathfrak{p} \cong \mathfrak{p}^{*}$ using a fixed $\mathrm{K}$-invariant inner product on $\mathfrak{g}$.) Consider the map $p: T^{*}(G / K) \rightarrow G / K$ given by $p([g, X])=g K$, for $g \in G$ and $X \in \mathfrak{p}$. As in (3.15), we have a G-equivariant decomposition of the tangent bundle

$$
\mathrm{TM} \cong \mathrm{p}^{*} \mathrm{~T}(\mathrm{G} / \mathrm{K}) \oplus \mathrm{G} \times \mathrm{K}(\mathrm{Tp}) .
$$

Consider the K-equivariant vector bundle $\varepsilon_{\mathfrak{p}}:=\mathfrak{p} \times S_{\mathfrak{p}} \rightarrow \mathfrak{p}$, and the Gequivariant vector bundle $\mathcal{E}_{\mathrm{G} / \mathrm{K}}:=\mathrm{G} \times{ }_{\mathrm{K}} S_{\mathfrak{p}} \rightarrow \mathrm{G} / \mathrm{K}$. Let us form

$$
\mathcal{E}_{M}:=p^{*} \varepsilon_{G / K} \otimes\left(G \times{ }_{K} \varepsilon_{p}\right),
$$

which defines a G-equivariant spinor bundle on $M$. Here, and in the remainder of this subsection, we use graded tensor products. Note that the bundle $\mathcal{E}_{M}$ contains two factors $S_{\mathfrak{p}}$, coming from the spinor bundles on $\mathrm{G} / \mathrm{K}$ and $\mathfrak{p}$. The natural Clifford actions by $T \mathfrak{p}$ on $\varepsilon_{\mathfrak{p}}$ and by $T(G / K)$ on $\mathcal{E}_{\mathrm{G} / \mathrm{K}}$ (both denoted by c) combine to a Clifford action

$$
c: T M \cong p^{*} T(G / K) \oplus G \times_{K}(T p) \rightarrow \operatorname{End}\left(\varepsilon_{M}\right),
$$

given by

$$
c(v, w)=c(v) \otimes 1+1 \otimes c(w),
$$

for $v \in p^{*} \mathrm{~T}(\mathrm{G} / \mathrm{K})$ and $w \in \mathrm{Tp}$.

Let $V \in \hat{R}$. Consider the $\mathrm{G}$-equivariant vector bundle

$$
\mathcal{E}_{\mathrm{V}}:=\mathcal{E}_{\mathrm{M}} \otimes\left(\mathrm{G} \times \times_{\mathrm{K}}(\mathfrak{p} \times \mathrm{V})\right) \rightarrow \mathrm{M} .
$$

By construction, we have

$$
\Gamma^{\infty}\left(\mathcal{E}_{\mathrm{V}}\right) \cong\left(\mathrm{C}^{\infty}(\mathrm{G}) \otimes \mathrm{S}_{\mathfrak{p}} \otimes \mathrm{V} \otimes \mathrm{C}^{\infty}\left(\mathfrak{p}, \mathrm{S}_{\mathfrak{p}}\right)\right)^{\mathrm{K}}
$$

Let $D_{\mathfrak{p}}$ be the K-equivariant Dirac operator on $C^{\infty}\left(\mathfrak{p}, S_{\mathfrak{p}}\right)$ so that

$$
\left[\mathrm{D}_{\mathfrak{p}}\right] \in \mathrm{KK}^{\mathrm{K}}\left(\mathrm{C}_{0}(\mathfrak{p}), \mathbb{C}\right)
$$

is the fundamental class. Let $\mathrm{D}_{\mathrm{V}}$ be the operator on

$$
C^{\infty}(G) \otimes S_{\mathfrak{p}} \otimes V
$$


defined as in (2.4). We take the Dirac operator $D_{M}^{V}$ on 4.6 to be

$$
\mathrm{D}_{\mathrm{M}}^{\mathrm{V}}:=\mathrm{D}_{\mathrm{V}} \otimes 1+1 \otimes \mathrm{D}_{\mathfrak{p}}
$$

Let $\psi: M \rightarrow \mathfrak{g}$ be the G-equivariant map defined by

$$
\psi[\mathrm{g}, X]=\operatorname{Ad}(\mathrm{g}) X,
$$

for $g \in G$ and $X \in \mathfrak{p}$. The vanishing set Zeroes $\left(v^{\psi}\right)$ is the zero section of the vector bundle $G \times_{K} \mathfrak{p} \rightarrow G / K$, and hence cocompact. Let $\rho \in C^{\infty}(M)^{G}$ be as in Theorem 2.1, and let $f \in C^{\infty}(M)^{G}$ be $\rho$-admissible. Consider the deformed Dirac operator

$$
D_{f \psi}^{V}:=D_{M}^{V}-\sqrt{-1} f c\left(v^{\psi}\right) .
$$

on $\mathcal{E}_{V}$. It has a G-index

$$
\operatorname{index}_{G}\left(\varepsilon_{V}, \psi\right) \in K K\left(C_{0}(G / K) \rtimes G, \mathbb{C}\right) .
$$

Proposition 4.9. Under the isomorphism $\mathrm{KK}\left(\mathrm{C}_{0}(\mathrm{G} / \mathrm{K}) \rtimes \mathrm{G}, \mathbb{C}\right) \cong \widehat{\mathrm{R}}(\mathrm{K})$ given by Morita equivalence, we have

$$
\operatorname{index}_{\mathrm{G}}\left(\varepsilon_{\mathrm{V}}, \psi\right)=[\mathrm{V}] \text {. }
$$

Let us prove this proposition. We denote by $\overline{S_{\mathfrak{p}}}=\mathfrak{p} \times \mathrm{S}_{\mathfrak{p}}$ the trivial vector bundle over $\mathfrak{p}$ with fibre $S_{\mathfrak{p}}$. Consider the vector bundle endomorphism $\beta$ of $\overline{S_{\mathfrak{p}}}$ given by

$$
\beta(X, \xi):=(X, c(X) \xi),
$$

for $X \in \mathfrak{p}$ and $\xi \in S_{\mathfrak{p}}$. It is invertible outside the compact set $\{0\} \subset \mathfrak{p}$, and hence defines a class in the (K-equivariant) topological K-theory of $\mathfrak{p}$. This is the Bott element, denoted by $[\beta]$.

We choose an orthonormal basis $\left\{X_{1}, \ldots, X_{\operatorname{dim} \mathfrak{p}}\right\}$ of $\mathfrak{p}$. Let $\left\{\eta_{1}, \ldots, \eta_{\operatorname{dim} \mathfrak{p}}\right\}$ be the dual basis of $\mathfrak{p}^{*}$. Then the identity map on $\mathfrak{p}$ equals $\sum_{j=1}^{\operatorname{dim} \mathfrak{p}} X_{\mathfrak{j}} \otimes \eta_{j}$, and we have

$$
\beta=\sum_{j=1}^{\operatorname{dim} \mathfrak{p}} c\left(X_{j}\right) \otimes \eta_{j} .
$$

Consider the operator

$$
\mathrm{D}_{\mathrm{f}}:=\mathrm{D}_{\mathfrak{p}} \otimes 1-1 \otimes \sqrt{-1} \mathrm{f} \cdot \beta
$$

on $\Gamma^{\infty}\left(\varepsilon_{\mathfrak{p}} \otimes \overline{S_{\mathfrak{p}}}\right)$, and the bounded operator

$$
F_{f}:=\frac{D_{f}}{\sqrt{1+D_{f}^{2}}}
$$

on $\mathrm{L}^{2}\left(\mathfrak{p}, \mathcal{E}_{\mathfrak{p}} \otimes \overline{\mathrm{S}_{\mathfrak{p}}}\right)$. 
Lemma 4.10. There is a real-valued function $\rho \in C^{\infty}(\mathfrak{p})^{\mathrm{K}}$, such that if $\mathrm{f}$ is $\rho$ admissible, the operator $\mathrm{F}_{\mathrm{f}}$ is Fredholm, and its index is given by the trivial $\mathrm{K}$ representation.

Proof. Since $\overline{S_{\mathfrak{p}}}$ is a trivial vector bundle over $\mathfrak{p}$, we have

$$
\mathrm{L}^{2}\left(\mathfrak{p}, \mathcal{E}_{\mathfrak{p}} \otimes \overline{S_{\mathfrak{p}}}\right) \cong \mathrm{L}^{2}\left(\mathfrak{p}, \mathcal{E}_{\mathfrak{p}}\right) \otimes S_{\mathfrak{p}}
$$

By (4.7), the operator $D_{f}$ can be rewritten as

$$
D_{\mathfrak{p}} \otimes 1-\sqrt{-1} \sum_{j=1}^{\operatorname{dim} \mathfrak{p}} f \eta_{j} \otimes \mathfrak{c}\left(X_{j}\right) .
$$

Therefore, $\left[D_{\mathfrak{p}}, f \beta\right] \in \operatorname{End}\left(\varepsilon_{\mathfrak{p}} \otimes \overline{S_{\mathfrak{p}}}\right)$, so that $D_{f}$ is an operator as in Remark 3.4. That remark therefore implies that there is a function $\rho$ such that $F_{f}$ is Fredholm if $f$ is $\rho$-admissible. Then the index of $F_{f}$ is the class

$$
\left[D_{\mathfrak{f}}\right]:=\left[\mathrm{L}^{2}\left(\mathfrak{p}, \mathcal{E}_{\mathfrak{p}} \otimes \overline{\mathrm{S}_{\mathfrak{p}}}\right), \mathrm{F}_{\mathfrak{f}}\right] \in \mathrm{KK}^{\mathrm{K}}(\mathbb{C}, \mathbb{C}) .
$$

To calculate this index, we write

$$
F_{\mathfrak{p}}:=\frac{D_{\mathfrak{p}}}{\sqrt{1+D_{\mathfrak{p}}^{2}}}
$$

We view $\Gamma_{0}\left(\overline{S_{\mathfrak{p}}}\right)$ as a Hilbert $C_{0}(\mathfrak{p})$-module, and consider the representation of $C_{0}(\mathfrak{p})$ in $\mathrm{L}^{2}\left(\mathfrak{p}, \mathcal{E}_{\mathfrak{p}}\right)$ given by pointwise multiplication. Then

$$
\mathrm{L}^{2}\left(\mathfrak{p}, \mathcal{E}_{\mathfrak{p}} \otimes \overline{S_{\mathfrak{p}}}\right) \cong \Gamma_{0}\left(\overline{S_{\mathfrak{p}}}\right) \otimes_{\mathrm{C}_{0}(\mathfrak{p})} \mathrm{L}^{2}\left(\mathfrak{p}, \mathcal{E}_{\mathfrak{p}}\right) .
$$

One can check that $F_{f}$ is an $F_{p}$-connection [23, Definition 2.6]. This implies that the element $\left[D_{f}\right] \in K^{K}(\mathbb{C}, \mathbb{C})$ is the Kasparov product over $C_{0}(\mathfrak{p})$ of the classes $[f \beta] \in K^{K}\left(\mathbb{C}, C_{0}(\mathfrak{p})\right)$ and $\left[D_{\mathfrak{p}}\right] \in \mathrm{KK}^{K}\left(\mathrm{C}_{0}(\mathfrak{p}), \mathbb{C}\right)$. (One can also find a proof in Lemma 3.1 in [25].)

Note that $f$ is a positive function. By a homotopy, we have $[f \cdot \beta]=[\beta]$. By Proposition 11.4.5 in [15], the Kasparov product of $\left[D_{\mathfrak{p}}\right]$ and $[\beta]$ is the trivial representation of $\mathrm{K}$.

Proof of Proposition 4.9. By Theorem 3.2, the Morita equivalence isomorphism maps index $\operatorname{men}_{\mathrm{G}}\left(\mathcal{E}_{\mathrm{V}}, \psi\right)$ to the K-index of the operator $\mathrm{D}_{\mathrm{f}} \otimes 1_{\mathrm{V}}$ on

$$
\mathrm{L}^{2}\left(\mathfrak{p}, \mathcal{E}_{\mathfrak{p}} \otimes \overline{\mathrm{S}_{\mathfrak{p}}}\right) \otimes \mathrm{V} .
$$

By Lemma 4.10, this index equals $[\mathrm{V}] \in \widehat{R}(\mathrm{~K})$.

Motivated by Proposition 4.9, we define a K-homological version of Dirac induction. 
Definition 4.11. For any $\mathrm{V} \in \hat{\mathrm{R}}$, the K-homological Dirac induction of $\mathrm{V}$ is the class

$$
\widehat{\mathrm{D}-\mathrm{Ind}}_{\mathrm{K}}^{\mathrm{G}}(\mathrm{V}):=\operatorname{index}_{\mathrm{G}}\left(\mathcal{E}_{\mathrm{V}}, \psi\right) \in \mathrm{KK}\left(\mathrm{C}_{0}(\mathrm{G} / \mathrm{K}) \rtimes \mathrm{G}, \mathbb{C}\right) .
$$

Proposition 4.9 implies that this is an isomorphism.

Theorem 4.12. K-homological Dirac induction defines an isomorphism

$$
\widehat{R}(\mathrm{~K}) \stackrel{\cong}{\rightrightarrows} \mathrm{KK}\left(\mathrm{C}_{0}(\mathrm{G} / \mathrm{K}) \rtimes \mathrm{G}, \mathbb{C}\right) .
$$

Of course, the fact that $\widehat{R}(K) \cong K K\left(C_{0}(G / K) \rtimes G, \mathbb{C}\right)$ is not new, or as deep as the Dirac induction isomorphism for K-theory. But we found it interesting that this isomorphism can be described in terms of the G-index for a non-cocompact action. For example, this implies that any element of $K K\left(C_{0}(G / K) \rtimes G, \mathbb{C}\right)$ can be realised as a $G$-index for such an action. Furthermore, in this class of examples, the image of $N=\mathfrak{p}$ under $\psi$ does not lie inside $k$. (Nor for any other choice of the map $M \rightarrow G / K$.) Examples with these properties are furthest removed from existing index theory.

Remark 4.13. Let $V=\mathbb{C}$ be the trivial representation of $K$. The map $\psi$ is a moment map for the standard symplectic form on $T^{*}(G / K)$. Hence, following [27, 29], one can interpret the G-index of $D_{f \psi}^{\mathbb{C}}$ as the geometric quantisation $\mathrm{Q}_{\mathrm{G}}\left(\mathrm{T}^{*}(\mathrm{G} / \mathrm{K})\right)$ of $\mathrm{T}^{*}(\mathrm{G} / \mathrm{K})$. By Propositions 2.10 in [21] and Proposition 4.9. one then has

$$
\mathrm{Q}_{\mathrm{G}}\left(\mathrm{T}^{*}(\mathrm{G} / \mathrm{K})\right)=\left[\mathrm{L}^{2}(\mathrm{G} / \mathrm{K}), 0, \pi_{\mathrm{C}_{0}(\mathrm{G} / \mathrm{K}) \rtimes \mathrm{G}}\right] \quad \in \mathrm{KK}\left(\mathrm{C}_{0}(\mathrm{G} / \mathrm{K}) \rtimes \mathrm{G}, \mathbb{C}\right) .
$$

This looks natural, especially since the representation of $G$ in $L^{2}(G / K)$ is

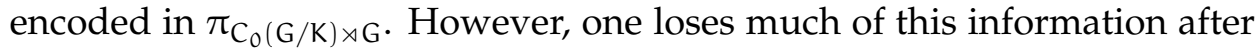
applying the homotopy relation in K-homology.

Example 4.14. Consider the situation of this subsection where $G=\mathbb{R}$, but we allow more general equivariant maps $\psi: M \rightarrow \mathfrak{g}$ than the one used above. Then we obtain an explicit example of how the G-index can depend on the map $\psi$.

In this case, the map $\psi: \mathbb{R}^{2} \rightarrow \mathbb{R}$ is of the form

$$
\psi(x, y)=\zeta(y)
$$

for a real-valued function $\zeta \in \mathrm{C}^{\infty}(\mathbb{R})$. The G-index of the Dirac operator deformed by $\psi$ now lies in

$$
\mathrm{KK}\left(\mathrm{C}_{0}(\mathbb{R}) \rtimes \mathbb{R}, \mathbb{C}\right) \cong \mathrm{KK}(\mathbb{C}, \mathbb{C}) \cong \mathbb{Z}
$$


It equals the difference of the dimensions of the spaces of square-integrable functions that are scalar multiples of the functions $s_{-}$and $s_{+}$on $\mathbb{R}$, respectively, defined by

$$
s_{ \pm}(y)=e^{ \pm \int_{0}^{y} \zeta(t) d t}
$$

for $y \in \mathbb{R}$.

Suppose that the function $\zeta$ is nowhere vanishing over $\mathbb{R}$, for example, $\zeta>0$ on $\mathbb{R}$. Then neither $s_{-}$nor $s_{+}$will be square-integrable. So the Gindex of the deformed Dirac operator equals zero. Note that the induced vector field $v^{\psi}$ is nowhere vanishing over $M$ in this case. If $\zeta(y)= \pm y$, then $s_{\mp}$ is square-integrable, but $s_{ \pm}$is not. It follows that the G-index of the deformed Dirac operator is equal to \pm 1 , with kernel spanned by the function $y \mapsto e^{-\frac{y^{2}}{2}}$ (in even or odd degree, respectively).

\section{4 $\operatorname{Spin}^{\mathrm{c}}$-quantisation commutes with reduction}

In this subsection, we consider the special case where D is a Spin ${ }^{c}$-Dirac operator. Suppose $G$ is reductive. Suppose $M$ is even-dimensional and has a G-equivariant $\operatorname{Spin}^{\mathrm{c}}$-structure. Let $\mathcal{E}$ be the associated spinor bundle. Furthermore, that $\mathrm{G} / \mathrm{K}$ is even-dimensional and equivariantly Spin. As before, let $S_{\mathfrak{p}} \in R(K)$ be the Spin representation of $\mathfrak{p}$. The Clifford module $\mathcal{E}_{N} \rightarrow N$ as in (3.10) is now the spinor bundle of a K-equivariant Spin $^{c}$-structure on N. (See Proposition 3.10 in [19].)

Let $L \rightarrow M$ be the determinant line bundle of the Spin ${ }^{c}$-structure. Then $\mathrm{L}_{\mathrm{N}}:=\left.\mathrm{L}\right|_{\mathrm{N}} \rightarrow \mathrm{N}$ is the determinant line bundle of the Spin ${ }^{\mathrm{c}}$-structure on $\mathrm{N}$ whose spinor bundle is $\varepsilon_{N}$. Let $\nabla^{\mathrm{L}_{N}}$ be a K-invariant, Hermitian connection on $\mathrm{L}_{\mathrm{N}}$. It defines a $\operatorname{Spin}^{\mathrm{c}}$-moment map

$$
\psi_{\mathrm{N}}: \mathrm{N} \rightarrow \mathfrak{k}^{*}
$$

by

$$
2 \sqrt{-1}\left\langle\psi_{N}, X\right\rangle=\mathcal{L}_{X}^{L_{N}}-\nabla_{X_{N}}^{L_{N}} \in \operatorname{End}\left(L_{N}\right)=C^{\infty}(N),
$$

for all $X \in \mathfrak{k}$. Here $\mathcal{L}^{L_{N}}$ is the Lie derivative of sections of $L_{N}$. In Section 3.1 of [16], a G-invariant, Hermitian connection $\nabla^{\mathrm{L}}$ on $\mathrm{L}$ is constructed, for which the associated $\operatorname{Spin}^{c}$-moment map $\psi: M \rightarrow \mathfrak{g}^{*}$ (defined analogously to $\psi_{\mathrm{N}}$ ), restricts to $\psi_{\mathrm{N}}$ on $\mathrm{N}$.

Fix a K-invariant inner product $(-,-)^{\mathfrak{g}}$ on $\mathfrak{g}$. We use this to identify $\mathfrak{k}^{*} \cong \mathfrak{k}$, and hence to view $\psi_{N}$ as a map into $\mathfrak{k}$. Furthermore, consider the trivial vector bundle $M \times \mathfrak{g} \rightarrow M$, on which $G$ act as

$$
g \cdot(m, X)=(g m, \operatorname{Ad}(g) X),
$$


for $\mathrm{g} \in \mathrm{G}, \mathrm{m} \in \mathrm{M}$ and $\mathrm{X} \in \mathfrak{g}$. We have a $\mathrm{G}$-invariant metric on this bundle, defined by

$$
(X, Y)_{g n}:=\left(\operatorname{Ad}\left(g^{-1}\right) X, \operatorname{Ad}\left(g^{-1}\right) Y\right)^{\mathfrak{g}},
$$

for $X, Y \in \mathfrak{g}, g \in G$ and $n \in N$. Using this to identify $M \times \mathfrak{g}^{*} \cong M \times \mathfrak{g}$, we view $\psi$ as a map into $\mathfrak{g}$.

Let $\rho, \rho_{N} \in C^{\infty}(M)^{G}$ be as in Theorems 2.1 and 3.2. Suppose $f \in$ $C^{\infty}(M)^{G}$ is $\max \left(\rho, \rho_{N}\right)$-admissible. Consider the deformed Dirac operator $D_{f \psi}$. We will see that the image of its G-index in $\widehat{R}(K)$ decomposes into irreducible representations according to the quantisation commutes with reduction principle. In the $\mathrm{Spin}^{\mathrm{c}}$-setting, this principle was first proved for compact groups and manifolds in [30]. This was generalised to noncompact manifolds, but still compact groups, in [20].

Let us make this precise. For any $\lambda, v$ in the set $\Lambda_{+}$of dominant weights used before, let $n_{\lambda}^{v}$ be the nonnegative integers such that

$$
V_{\lambda} \otimes S_{\mathfrak{p}}=\bigoplus_{\nu \in \Lambda_{+}} n_{\lambda}^{v} V_{v}
$$

For $\lambda \in \Lambda_{+}$, let

$$
M_{\lambda}:=\psi^{-1}(\lambda / \sqrt{-1}) / G_{\lambda},
$$

be the reduced space at $\lambda$, where $G_{\lambda}<G$ is the stabiliser of $\lambda$ with respect to the coadjoint action. Since $G$ is reductive, we have

$$
M_{\lambda}=N_{\lambda}:=\psi_{N}^{-1}(\lambda / \sqrt{-1}) / K_{\lambda} .
$$

(See Proposition 3.13 in [19].)

Suppose $\psi$ is G-proper, in the sense that the inverse image of a cocompact set is cocompact. Then $M_{\lambda}$ is compact. This space has a Spin ${ }^{c}-$ quantisation

$$
\mathrm{Q}\left(\mathrm{N}_{\lambda}\right) \in \mathbb{Z},
$$

as defined in Section 5 of [30]. In the sufficiently regular case, this is the index of a Spin ${ }^{c}$-Dirac operator with respect to a Spin ${ }^{c}$-structure induced by the one on $\mathrm{N}$. Then this index equals the index of a corresponding Spin ${ }^{\mathrm{c}}-$ Dirac operator on $M_{\lambda}$ (see Proposition 3.14 in [19]). It therefore makes sense to define

$$
\mathrm{Q}\left(\mathrm{M}_{\lambda}\right):=\mathrm{Q}\left(\mathrm{N}_{\lambda}\right) .
$$


Corollary 4.15 (Spin $^{c}$-quantisation commutes with reduction). Suppose that $\mathrm{G}$ is reductive, and that $\mathrm{G} / \mathrm{K}$ is even-dimensional and equivariantly Spin. Consider the multiplicities $\mathrm{m}_{\lambda} \in \mathbb{Z}$ in

$$
\operatorname{index}_{G}(\varepsilon, \psi)=\sum_{\lambda \in \Lambda_{+}} m_{\lambda} V_{\lambda} \in \widehat{R}(K) \cong K K\left(C_{0}(G / K) \rtimes G, \mathbb{C}\right) .
$$

If the action by $\mathrm{G}$ on $\mathrm{M}$ has Abelian stabilisers on an open dense subset of $\mathrm{M}$, then for all $\lambda \in \Lambda_{+}$,

$$
\mathrm{m}_{\lambda}=\sum_{v \in \Lambda_{+}} \mathrm{n}_{\lambda}^{v} \mathrm{Q}\left(\mathrm{M}_{v+\rho_{\mathrm{K}}}\right)
$$

In general, without an assumption on the stabilisers, we have

$$
\mathrm{m}_{\lambda}=\sum_{\nu \in \Lambda_{+}} \mathrm{n}_{\lambda}^{v}\left(\sum_{j=1}^{k_{\lambda}} \mathrm{Q}\left(\mathrm{M}_{\nu+\rho_{\mathrm{j}}}\right)\right),
$$

for a finite set $\left\{\rho_{1}, \ldots, \rho_{\mathrm{k}_{\lambda}}\right\} \subset i \mathrm{t}^{*}$, as specified in Theorem $1.4 \mathrm{in}$ [30].

Proof. We saw that in this case, $\psi(N) \subset \mathfrak{k}$. Therefore, Corollary 3.8 implies that

$$
\begin{aligned}
m_{\lambda} & =\operatorname{dim}\left(\operatorname{index}_{K} D_{f \psi}^{\varepsilon_{N}} \otimes S_{p} \otimes V_{\lambda}^{*}\right)^{K} \\
& =\sum_{v \in \Lambda_{+}} n_{\lambda}^{v} \operatorname{dim}\left(\operatorname{index}_{K} D_{f \psi}^{\varepsilon_{N}} \otimes V_{v}^{*}\right)^{K} .
\end{aligned}
$$

Here we used the fact that $S_{\mathfrak{p}}^{*}=S_{\mathfrak{p}}$. By Theorem 3.9 in [20], the multiplicity

$$
\operatorname{dim}\left(\operatorname{index}_{K} D_{f \psi}^{\mathcal{E}_{N}} \otimes V_{v}^{*}\right)^{K}
$$

is given by the desired expression.

Remark 4.16. We always assumed that Zeroes $\left(v^{\psi}\right) / \mathrm{G}$ was compact. In [20], however, it was explained how to handle the case where this condition does not hold, if $\mathrm{G}$ is compact. Via Corollary 3.8, the same methods apply to noncompact $\mathrm{G}$. Therefore, there is still a well-defined index, and Corollary 4.15 still holds, if Zeroes $\left(v^{\psi}\right) / \mathrm{G}$ is noncompact. It is still essential that $\psi$ is G-proper. 


\section{A Notation and conventions}

- If $X$ is a locally compact Hausdorff space, then $C(X)$ is the algebra of continuous, complex valued functions on $X$. We will mention explicitly where we assume a function to be real-valued. We write $C_{b}(X)$, $\mathrm{C}_{0}(\mathrm{X})$ and $\mathrm{C}_{\mathrm{c}}(\mathrm{X})$ for the algebras of bounded functions, functions vanishing at infinity, and compactly supported functions in $C(X)$, respectively. The sup-norm of a function $f \in C_{b}(X)$ is denoted by $\|f\|_{\infty}$.

- If $\mathcal{E} \rightarrow \mathrm{X}$ is a vector bundle (tacitly assumed to be continuous), then $\Gamma(\mathcal{E})$ is the space of continuous sections of $\mathcal{E}$, and $\Gamma_{\mathcal{c}}(\mathcal{E}) \subset \Gamma(\mathcal{E})$ is the subspace of compactly supported sections. If $\mathcal{E}$ is equipped with a metric, then $\Gamma_{0}(\mathcal{E})$ is the space of continuous sections vanishing at infinity. If, in addition, $X$ is equipped with a Borel measure, then $\mathrm{L}^{2}(\varepsilon)$ is the space of $\mathrm{L}^{2}$-sections of $\mathcal{E}$. If $\mathcal{E}^{\prime} \rightarrow \mathrm{X}^{\prime}$ is another vector bundle, then $\varepsilon \otimes \mathcal{E}^{\prime} \rightarrow X \times X^{\prime}$ is the exterior tensor product of the two.

- Analogously, if $M$ is a smooth manifold, we have the algebras $C^{\infty}(M)$, $\mathrm{C}_{\mathrm{b}}^{\infty}(M), \mathrm{C}_{0}^{\infty}(M)$ and $\mathrm{C}_{\mathrm{c}}^{\infty}(M)$ of smooth, complex valued functions on $M$ with the various growth/decay properties towards infinity. The tangent bundle projection of $M$ is denoted by $\tau_{M}$ : TM $\rightarrow M$. The space of smooth vector fields on $M$ is denoted by $X(M)$. The set of zeroes of a vector field $v \in X(M)$ is denoted by Zeroes $(v)$. If $\mathcal{E} \rightarrow M$ is a (smooth) vector bundle, then we have the space $\Gamma^{\infty}(\varepsilon)$ of smooth sections, and its subspace $\Gamma_{\mathrm{c}}^{\infty}(\varepsilon)$ of compactly supported sections.

- If $M$ has a Riemannian metric, we will use it to identify $T^{*} M \cong T M$. We denote the Levi-Civita connection on TM by $\nabla^{\mathrm{TM}}$. If, in addition, $\mathcal{E}$ has a metric, the space $\mathrm{L}^{2}(\mathcal{E})$ will be defined with respect to the Riemannian density.

\section{References}

[1] Herbert Abels. Parallelizability of proper actions, global K-slices and maximal compact subgroups. Math. Ann., 212:1-19, 1974/75.

[2] Dmitri Alekseevsky, Andreas Kriegl, Mark Losik, and Peter W. Michor. The Riemannian geometry of orbit spaces-the metric, geodesics, and integrable systems. Publ. Math. Debrecen, 62(3-4):247-276, 2003. Dedicated to Professor Lajos Tamássy on the occasion of his 80th birthday.

[3] Nicolae Anghel. On the index of Callias-type operators. Geom. Funct. Anal., 3(5):431-438, 1993. 
[4] Michael Atiyah. Elliptic operators and compact groups. Lecture Notes in Mathematics, Vol. 401. Springer-Verlag, Berlin, 1974.

[5] Dan Barbasch and Henri Moscovici. $\mathrm{L}^{2}$-index and the Selberg trace formula. J. Funct. Anal., 53(2):151-201, 1983.

[6] Paul Baum, Alain Connes, and Nigel Higson. Classifying space for proper actions and K-theory of group C*-algebras. In C*-algebras: 1943-1993 (San Antonio, TX, 1993), volume 167 of Contemp. Math., pages 240-291. American Mathematical Society, Providence, RI, 1994.

[7] Bruce Blackadar. K-theory for operator algebras, volume 5 of Mathematical Sciences Research Institute Publications. Cambridge University Press, Cambridge, second edition, 1998.

[8] Nicolas Bourbaki. Éléments de mathématique. Fascicule XXIX. Livre VI: Intégration. Chapitre 7: Mesure de Haar. Chapitre 8: Convolution et représentations. Actualités Scientifiques et Industrielles, No. 1306. Hermann, Paris, 1963.

[9] Maxim Braverman. Index theorem for equivariant Dirac operators on noncompact manifolds. K-Theory, 27(1):61-101, 2002.

[10] Maxim Braverman. The index theory on non-compact manifolds with proper group action. J. Geom. Phys., 98:275-284, 2015.

[11] Jochen Brüning and Henri Moscovici. $\mathrm{L}^{2}$-index for certain Dirac-Schrödinger operators. Duke Math. J., 66(2):311-336, 1992.

[12] Ulrich Bunke. A K-theoretic relative index theorem and Callias-type Dirac operators. Math. Ann., 303(2):241-279, 1995.

[13] Constantine Callias. Axial anomalies and index theorems on open spaces. Comm. Math. Phys., 62(3):213-234, 1978.

[14] Jérôme Chabert, Siegfried Echterhoff, and Ryszard Nest. The ConnesKasparov conjecture for almost connected groups and for linear p-adic groups. Publ. Math. Inst. Hautes Études Sci., (97):239-278, 2003.

[15] Nigel Higson and John Roe. Analytic K-homology. Oxford Mathematical Monographs. Oxford University Press, Oxford, 2000. Oxford Science Publications.

[16] Peter Hochs. Quantisation commutes with reduction at discrete series representations of semisimple groups. Adv. Math., 222(3):862-919, 2009.

[17] Peter Hochs. Decomposing equivariant indices of Spin ${ }^{c}$-Dirac operators for cocompact actions. ArXiv:1412.5348, 2014.

[18] Peter Hochs and Varghese Mathai. Geometric quantization and families of inner products. Adv. Math., 282:362-426, 2015.

[19] Peter Hochs and Varghese Mathai. Quantising proper actions on Spin ${ }^{\mathrm{c}}$ manifolds. Asian J. Math., (to appear), 2016. ArXiv:1408.0085. 
[20] Peter Hochs and Yanli Song. Equivariant indices of Spin ${ }^{c}$-Dirac operators for proper moment maps. ArXiv:1503.00801, 2015.

[21] Peter Hochs and Yanli Song. An equivariant index for proper actions I: construction of the index. ArXiv:1512.07575, 2016.

[22] Gennadi Kasparov. Index of invariant elliptic operators, K-theory and representations of Lie groups. Dokl. Akad. Nauk SSSR, 268(3):533-537, 1983.

[23] Gennadi Kasparov. Equivariant KK-theory and the Novikov conjecture. Invent. Math., 91(1):147-201, 1988.

[24] Gennadi Kasparov. K-theoretic index theorems for elliptic and transversally elliptic operators. J. Noncommut. Geom., 2016. Version November 2013.

[25] Dan Kucerovsky. A short proof of an index theorem. Proc. Amer. Math. Soc., 129(12):3729-3736, 2001.

[26] H. Blaine Lawson, Jr. and Marie-Louise Michelsohn. Spin geometry, volume 38 of Princeton Mathematical Series. Princeton University Press, Princeton, NJ, 1989.

[27] Xiaonan Ma and Weiping Zhang. Geometric quantization for proper moment maps: the Vergne conjecture. Acta Math., 212(1):11-57, 2014.

[28] Varghese Mathai and Weiping Zhang. Geometric quantization for proper actions. Adv. Math., 225(3):1224-1247, 2010. With an appendix by Ulrich Bunke.

[29] Paul-Émile Paradan. Formal geometric quantization II. Pacific J. Math., 253(1):169-211, 2011.

[30] Paul-Émile Paradan and Michèle Vergne. Equivariant Dirac operators and differentiable geometric invariant theory. ArXiv:1411.7772, 2014.

[31] Rajagopalan Parthasarathy. Dirac operator and the discrete series. Ann. of Math. (2), 96:1-30, 1972.

[32] Marc Rieffel. Applications of strong Morita equivalence to transformation group C*-algebras. In Operator algebras and applications, Part I (Kingston, Ont., 1980), volume 38 of Proc. Sympos. Pure Math., pages 299-310. American Mathematical Society, Providence, RI, 1982.

[33] Antony Wassermann. Une démonstration de la conjecture de ConnesKasparov pour les groupes de Lie linéaires connexes réductifs. C. R. Acad. Sci. Paris Sér. I Math., 304(18):559-562, 1987.

[34] Dana Williams. Crossed products of $\mathrm{C}^{*}$-algebras, volume 134 of Mathematical Surveys and Monographs. American Mathematical Society, Providence, RI, 2007. 